\title{
Prediction of transmission, reflection and absorption coefficients of periodic structures using a hybrid Wave Based - Finite Element unit cell method
}

\author{
Elke Deckers $^{\mathrm{a}, \mathrm{b}, *}$, Stijn Jonckheere ${ }^{\mathrm{a}, \mathrm{b}, \mathrm{c}}$, Lucas Van Belle ${ }^{\mathrm{a}, \mathrm{b}}$, Claus Claeys ${ }^{\mathrm{a}, \mathrm{b}}$, Wim Desmet , $^{\mathrm{a}, \mathrm{b}}$ \\ ${ }^{a}$ KU Leuven - Dept. of Mechanical Engineering, Celestijnenlaan 300B - box 2420, B-3001 Leuven, Belgium \\ ${ }^{b}$ Member of Flanders Make \\ ${ }^{c}$ Siemens Industry Software NV, Digital Factory, Product Lifecycle Management - Simulation and Test Solutions, Interleuvenlaan 68, B-3001 \\ Leuven, Belgium
}

\begin{abstract}
This paper presents a hybrid Wave Based Method - Finite Element unit cell method to predict the absorption, reflection and transmission properties of arbitrary, two-dimensional periodic structures. The planar periodic structure, represented by its unit cell combined with Bloch-Floquet periodicity boundary conditions, is modelled within the Finite Element Method, allowing to represent complex geometries and to include any type of physics. The planar periodic structure is coupled to semi-infinite acoustic domains above and/or below, in which the dynamic pressure field is modelled with the Wave Based Method, applying a wave function set that fulfills the Helmholtz equation and satisfies the Sommerfeld radiation condition and the Bloch-Floquet periodicity conditions inherently. The dynamic fields described within both frameworks are coupled using a direct coupling strategy, accounting for the mutual dynamic interactions via a weighted residual formulation. The method explicitly accounts for the interaction between the unit cell and the surrounding acoustic domain, also accounting for higher order periodic waves. The convergence of the method is analysed and its applicability is shown for a variety of problems, proving it to be a useful tool combining the strengths of two methods.

Keywords: periodic structures, absorption, reflection, transmission, Wave Based Method, Finite Element Method, unit cell, Bloch-Floquet
\end{abstract}

\section{Introduction}

Enhancing the vibro-acoustic performance of compact lightweight material systems is of main importance for many applications in aerospace, machine design, building acoustics and automotive industry [1], mainly driven by the crossing of two trends. On the one hand, there is an increasing awareness of the negative health impact of excessive noise and vibration exposure [2]. On the other hand, every unit of mass and volume removed from the logistics chain has a direct economic and ecological benefit. However, classic noise control engineering solutions typically come with

\footnotetext{
${ }^{*}$ Corresponding author, Tel.:+32 163726 79; Fax: +32 1632 29 87; http://www.mech.kuleuven.be/ E-mail address: elke.deckers@kuleuven.be.
} 
significant and unwanted mass and/or volume additions. In view of meeting the conflicting design requirements of low mass and compactness and good noise and vibration insulation or absorption performance, novel material concepts are needed, many of which rely on or exploit effects related to periodicity.

Poro-elastic materials are also often applied in noise control applications given their low cost and good sound absorption properties. To have an effective solution, as a rule of thumb, the thickness of the material applied should be at least a quarter of the wavelength, when backed by a rigid wall. This leads to bulky solutions in the low-frequency range. Recent solutions to improve the low-frequency absorption comprise amongst others double porosity materials, e.g. [3], providing a backing of the porous layer by periodic irregularities [4], inclusions in foams e.g. [5, 6], which may be embedded resonators $[7,8,9]$ to act on dedicated frequency bands dictated by the local resonators or combinations of the improvements above, e.g. see [10].

Locally resonant metamaterials have emerged in recent years showing great potential for noise and vibration insulation and control $[11,12,13,14]$. These metamaterials can achieve greatly improved noise and vibration attenuation in targeted and tunable frequency ranges, so called stopbands, which are frequency ranges in which no free wave propagation is possible. These stopbands are obtained by adding elastic resonant structures to an elastic host structure on a subwavelength scale, leading to a Fano-type interference between incoming and re-radiated cells around their resonance frequency [15]. By targeting the acoustically relevant out-of-plane flexural waves, a zone of strongly reduced sound transmission can be obtained $[16,17,18,19]$. Membrane-type metamaterial realisations for acoustic purposes rely on added masses in a subwavelength grid on thin membranes, obtaining maxima in sound transmission loss (STL) when the surface averaged membrane velocity is zero [20]. Thin elastic membranes have been combined with rigid platelets obtaining excellent absorption due to high damping related to the flapping motion of the platelets [21]. Also acoustic resonances can be used in acoustic metamaterial applications [22], exploiting complex shapes, such as [23, 24]. Besides, acoustic resonant metasurfaces, consisting of acoustic resonators structured on a surface in a regular grid, are also applied to obtain improved sound absorption, e.g. see [25, 26, 27].

Other recent solutions aim, amongst others, to improve microslit materials by using the lessons learnt from double porosity materials [28], to improve porous materials absorption by adding periodic rigid partitions leading to quarter wavelength resonators [29] and to combine structural metamaterials designed for improved STL with visco-thermal damping mechanisms resulting from microslits [30]. Promising, thin sound absorbing solutions have been studied exploiting slow sound, resulting from quarter wavelength resonators loaded by a.o. detuned quarter wavelength or Helmholtz resonators, with high dispersion [31, 32, 33, 34].

From the overview given above, it becomes clear that novel acoustic material design becomes more and more complex, involving detailed and complex geometries and multiphysics interactions. Emerging possibilities in additive manufacturing allow to create ever more complex structures that can have improved vibro-acoustic properties [35] or serve to realise the aforementioned novel solutions: For instance selective laser sintering has been used in [16], stereolithography in [32]. To support the above mentioned complex designs, numerical prediction tools are of high importance. In order to predict the outcome of a complex design without experimental trial and error, to validate the 
effect of design changes and to run optimisation studies. The recent solutions, mentioned above, bare in common that they often rely on periodicity, or can be modelled as such, i.e. composed of a repetition of a unit cell (UC). In order to describe the dynamic performance of periodic materials, classically infinite periodic media are considered, as they can be efficiently represented by their UC in combination with Bloch-Floquet periodicity conditions [36, 37].

A common way to predict the vibro-acoustic performance of infinite periodic materials, consists in calculating the dispersion curves, describing the wave propagation throughout the infinite periodic medium, based on its representative UC. By comparing these dispersion curves to the dispersion curves of air, the influence on the acoustic radiation can be predicted by assessing the occurrence of acoustic coincidence [38, 39]. A prediction of actual levels of absorption coefficient and STL is, however, not possible in this case, as the coupling between the acoustic surrounding and the periodic structure is not accounted for.

In the case of simple thin plates and mass-spring resonators, analytical wave approaches can be used to predict the STL, [17, 40, 41], however, for more complex geometries, numerical simulation techniques are needed to predict the vibro-acoustic performance. Recently, the Transfer Matrix Method (TMM) [42], an efficient and widely applied tool to model plane acoustic fields in layered media of infinite lateral dimensions, has been extended to account for periodicity conditions [43]. This method is very effective, but breaks down when higher order acoustic Bloch-Floquet modes have to be accounted for. The Wave Based Method (WBM) [44] and the Multipole Method [45], have been applied to predict absorption, reflection and transmission coefficients of porous and poro-elastic layers with periodic inclusions, explicitly accounting for the vibro-acoustic coupling between the UC and the surrounding semi-infinite acoustic domains, however, yet only apply to relatively simple geometries and are validated so far only for twodimensional simulations. The Finite Element Method [46] allows to model a unit cell involving a high geometrical complexity and any type of physics involved. In a pure FEM setting, the semi-infinite acoustic domains have to be truncated, which can be achieved by applying absorbing boundary conditions like the Dirichlet-to-Neumann (DtN) [47], infinite elements [48], and absorbing elements, such as the Perfectly Matched Layer (PML) [49, 50, 51]. Often, the latter is applied as it is readily available in software packages and allows to also introduce periodicity conditions on the semi-infinite acoustic domains, see e.g. [29, 52, 34]. Care has to be taken, however, as a PML may introduce an artificial amount of damping and is not well suited for periodic structures with Bloch-waves and the long wave limit where wavelengths are much larger than the UC considered [53, 5].

To analyse the vibro-acoustic response of periodic materials consisting of arbitrarily complex UCs, this paper proposes a hybrid Wave Based - Finite Element Unit Cell model as an extension of the WBM [44], also towards three-dimensional applications. The dynamic fields within the bounded UC are modelled with the Finite Element Method (FEM), allowing a high geometrical flexibility and arbitrary physics to be included. The planar periodic structure is coupled to semi-infinite acoustic domains above and/or below. The acoustic pressure fields inside the semi-unbounded acoustic domains are modelled with the WBM [54, 55], which is an indirect Trefftz approach [56]. In this paper, its approximation functions are formulated to inherently fulfill the Helmholtz equation, the Bloch-Floquet periodicity boundary conditions and the Sommerfeld radiation condition, not relying on any artificial truncation or 
discretisation of the domain, as would be required in a pure FEM setting. The dynamic field variables of both methods are coupled at the interface, using a direct coupling approach. Bloch-Floquet periodicity conditions are applied on the FE system matrices and the WB-FE coupling matrices. The method is similar as the one proposed in [5], however, the following differences apply: in [5] the method is only applied for the Helmholtz equation within the unit cell and not for general physics. Besides, the periodicity conditions are included in the weak form of the equation, following [57], imposing a periodic pressure field and demodulated test functions, which requires the weak form to be derived and implemented for each type of physics. In the current paper, the Bloch-Floquet boundary conditions are imposed via the boundary conditions of the UC, as proposed in [58], and as such the original FE equations and matrices can readily be used.

This paper is structured as follows. Section 2 introduces the mathematical problem description of an in-plane periodic structure, composed of different physical subdomains. Section 3 briefly recalls the modelling procedures of the FEM and the WBM and derives a 3D, periodic, semi-infinite acoustic wave function basis. The strengths and weaknesses of both methods are discussed and the need for a hybrid methodology is highlighted. The hybrid coupling framework, the application of the periodicity conditions to the FE and the coupling matrices and the applied three-step procedure to solve the system of equations are discussed. The method is verified for three different numerical cases in Section 4: (i) a flat multilayer system to benchmark the accuracy of the hybrid method against an analytical available solution, (ii) a porous domain with a rigid periodic inclusions using a WB reference, and (iii) a metamaterial plate to show the potential of the method to predict the response of lightweight systems with complex dynamics. Conclusions are given in Section 5 .

\section{Problem description}

This section briefly describes the mathematical problem setting, as represented schematically in Fig. 1. Timeharmonic motion with $e^{\mathrm{j} \omega t}$-dependence is assumed, where $\mathrm{j}$ is the imaginary unit $\left(\mathrm{j}^{2}=-1\right), \omega$ the circular frequency and $t$ the time.

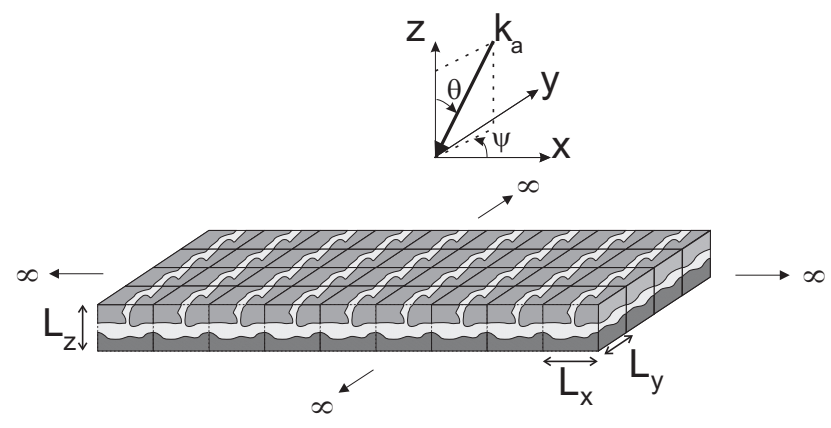

Figure 1: General problem description

An in-plane, infinite periodic structure is considered, coupled to one or two semi-infinite acoustic domains above 
and/or below. It is assumed that the interfaces with the semi-infinite acoustic domains are flat, which can always be achieved by adding an acoustic subdomain to the in-plane periodic structure. The thickness of the periodic structure is denoted $L_{z}$ and its spatial periodicity in the $x y$-plane is given by $L_{x}$ and $L_{y}$. The periodic structure is excited by an impinging acoustic plane wave with amplitude $A$ and wave number $\mathbf{k}_{\mathbf{a}}$, incident at elevation $\theta$, measured from the positive $z$-axis, and azimuth $\psi$, measured counterclockwise from the positive $x$-axis.

The semi-infinite acoustic domain is governed by the acoustic Helmholtz equation. This is complemented with the Sommerfeld radiation boundary condition which ensures that no acoustic energy is reflected at infinity. More details about the acoustc Helmholtz equation and the Sommerfeld radiation condition can be found in Section 3.1.

The in-plane periodic structure may be built up of any combination of physical subdomains for vibro-acoustic analysis. In the application cases of this paper, the following physical subdomains are considered:

- Acoustic or equivalent fluid domain $\Omega^{a}$ : described by the inhomogeneous Helmholtz equation, to which any equivalent fluid model of interest can be applied.

- Elastic solid domain $\Omega^{e}$ : described by the second-order Navier-Cauchy equation [59].

- Thin plate bending domain $\Omega^{b}$ : described by the Reissner-Mindlin equations [60, 61] .

- Poro-elastic domain $\Omega^{p}$ : described by the Biot poro-elasticity equations in the (u, $\left.p\right)$ - formulation [62, 63].

An overview of common Neumann, Dirichlet and mixed boundary conditions for the different physics considered and continuity conditions to ensure the coupling between different domains can be found in [64]. At the interface(s) with the periodic structure, coupling conditions need to be applied relating the dynamic fields within the semi-infinite acoustic domain(s) and the bounded adjacent domain(s) of the in-plane periodic structure. These coupling conditions are dependent on the physics involved in the periodic structure [64].

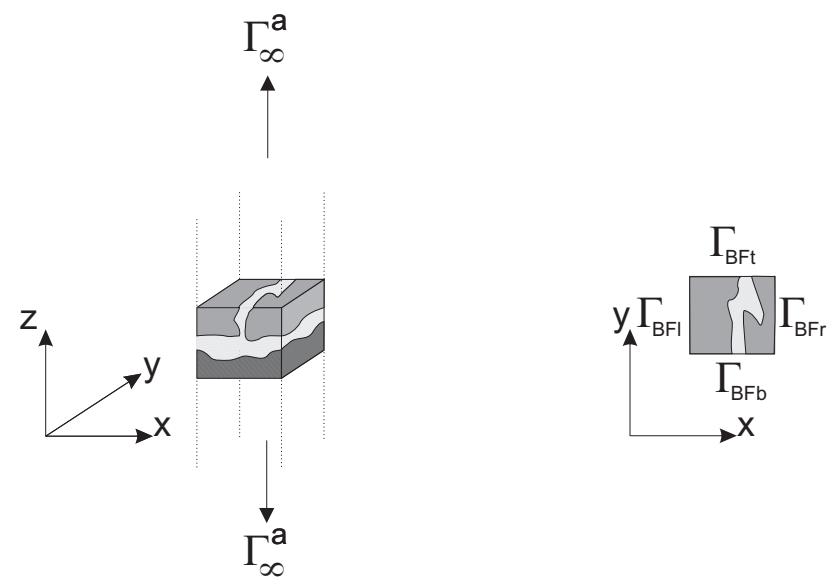

Figure 2: Left: general problem setting of a unit cell in a transmission context, right: top view on the UC with an indication of the boundaries

Independent of the physics involved in the periodic structure, due to the geometrical periodicity and the plane wave nature of the excitation, the resulting dynamic fields in the structure and in the semi-unbounded acoustic domains, 
have to be periodic in the $x$ - and $y$-direction. It is thus sufficient to obtain the dynamic behaviour of the entire infinite periodic structure, by determining the dynamic field $\zeta(x, y, z)$ in a single reference $\mathrm{UC}$, as shown in Fig. 2, and using the Bloch-Floquet periodicity relations [36, 37]:

$$
\forall M, N \in \mathbb{Z}, \zeta\left(x+M L_{x}, y+N L_{y}, z\right)=\zeta(x, y, z) e^{-\mathrm{j}\left(k_{a x} N L_{x}+k_{a y} M L_{y}\right)}
$$

where $x$ and $y$ are limited to positions in the reference $\mathrm{UC}, k_{a x}=-k_{a} \sin \theta \cos \psi$ and $k_{a y}=-k_{a} \sin \theta \sin \psi$ and $M$ and $N$ are integers positioning the $\mathrm{UC}$ of interest relative to the reference $\mathrm{UC}$ along the $x$ - and $y$-directions respectively.

\section{Numerical model}

In this paper, the Finite Element Method [46, 65] is used to model the dynamic field variables in the bounded unit cell of the in-plane periodic structure, allowing for an arbitrary geometrical complexity and to include any physics of interest. The dynamic pressure field in the semi-unbounded domain(s), is approximated using the WBM. The latter allows to inherently account for the Sommerfeld radiation condition in the semi-unbounded acoustic domains and the periodicity conditions of Eq. (1) and straightforwardly obtain expressions for the reflection, transmission and absorption coefficient of the periodic structure for given angles of incidence. A direct coupling of both methodologies into a direct hybrid approach is adopted.

In what follows, Section 3.1 describes the WBM and details the introduction of the periodicity conditions for the semi-unbounded domain(s). Section 3.2 highlights the need for a hybrid methodology, describes the hybrid coupling approach, details the application of the periodicity conditions on the FEM part and the coupling matrices and gives the three-step solution procedure of the hybrid system of equations.

\subsection{The WBM modelling procedure for $3 D$ semi-infinite periodic domains}

The WBM [54,55] is a numerical prediction technique which is based on an indirect Trefftz approach [56], approximating the dynamic field variables as a weighted expansion of wave functions which inherently satisfy the governing equation, in this case the Helmholtz :

$$
\nabla^{2} p^{a}(\mathbf{r})+k_{a}^{2} p^{a}(\mathbf{r})=\mathcal{F}_{a}(\mathbf{r})
$$

Boundary and interface residuals are minimised in a weighted residual approach, yielding a linear system of equations which can be solved for the contribution factors of each of the wave functions. For the unit cell shown in Fig. 2, the following boundary and continuity conditions apply on the boundaries of the semi-unbounded acoustic domains:

- Sommerfeld radiation condition: On the boundary at infinity, indicated $\Gamma_{\infty}^{a}$ (see Fig. 2), the Sommerfeld radiation condition for outgoing waves applies, ensuring that no acoustic energy is reflected back [66]:

$$
\mathbf{r} \in \Gamma_{\infty}^{a}: R_{\infty}^{a}(\mathbf{r})=\lim _{|\mathbf{r}| \rightarrow \infty}|\mathbf{r}|\left(\frac{\partial p^{a}}{\partial|\mathbf{r}|}-\mathrm{j} k_{a} p^{a}\right)=0
$$


- Bloch-Floquet periodicity conditions: The Bloch-Floquet periodicity conditions on acoustic pressure and normal velocity have to be imposed between the left, $\Gamma_{B F l}$, and right, $\Gamma_{B F r}$, and bottom, $\Gamma_{B F b}$ and top boundary, $\Gamma_{B F t}$, of the unit cell, respectively, see Fig. 2, leading to the following residuals:

$$
\begin{array}{ll}
\mathbf{r}^{\mathbf{l}} \in \Gamma_{B F l}^{a}: & R_{B F l}(\mathbf{r})=p^{a}\left(\mathbf{r}^{\mathbf{l}}\right)-p^{a}\left(\mathbf{r}^{\mathbf{r}}\right) e^{\mathrm{j} k_{a x} L_{x}}=0, \\
\mathbf{r}^{\mathbf{r}} \in \Gamma_{B F r}^{a}: & R_{B F r}(\mathbf{r})=\mathcal{L}_{v}\left(p^{a}\left(\mathbf{r}^{\mathbf{l}}\right)\right)+\mathcal{L}_{v}\left(p^{a}\left(\mathbf{r}^{\mathbf{l}}\right)\right) e^{-\mathrm{j} k_{a x} L_{x}}=0, \\
\mathbf{r}^{\mathbf{b}} \in \Gamma_{B F b}^{a}: & R_{B F b}(\mathbf{r})=p^{a}\left(\mathbf{r}^{\mathbf{b}}\right)-p^{a}\left(\mathbf{r}^{\mathbf{t}}\right) e^{\mathrm{j} k_{a y} L_{y}}=0, \\
\mathbf{r}^{\mathbf{t}} \in \Gamma_{B F t}^{a}: & R_{B F t}(\mathbf{r})=\mathcal{L}_{v}\left(p^{a}\left(\mathbf{r}^{\mathbf{t}}\right)\right)+\mathcal{L}_{v}\left(p^{a}\left(\mathbf{r}^{\mathbf{b}}\right)\right) e^{-\mathrm{j} k_{a y} L_{y}}=0 .
\end{array}
$$

The velocity operator is defined as:

$$
\mathcal{L}_{v}(\bullet)=\frac{\mathrm{j}}{\rho_{a} \omega} \frac{\partial \bullet}{\partial \gamma_{\mathbf{n}}},
$$

with $\gamma_{\mathbf{n}}$ the normal direction to the boundary, pointing outwards.

- Coupling conditions to the unit cell: These conditions boil down to a stress equilibrium between the acoustic pressure field and the stresses of the subdomain of the unit cell on the interfaces and a continuity of flux. They thus depend on the physics involved in the unit cell.

The general modelling procedure consists of the following four steps, which will be briefly discussed afterwards for the specific case of a periodic, semi-infinite acoustic domain:

1. Partitioning of the problem domain into convex subdomains,

2. Selection of the wave functions in the pressure expansion within each subdomain,

3. Construction of the system of equations via a weighted residual formulation of the boundary conditions and the continuity conditions,

4. Solution of the system of equations and postprocessing of the dynamic variables.

\subsubsection{Partitioning of the problem domain into subdomains}

A sufficient condition for convergence of the WBM is that the considered subdomains are convex [54], which is inherently fulfilled for the semi-unbounded domains considered, given that its interface with the FE unit cell is parallel to the $x y$-plane. The different WB acoustic subdomains are indicated as $\Omega^{(\alpha)}$, where $\alpha=1$ for an absorption problem and $\alpha \in\{1,2\}$ for a transmission problem.

\subsubsection{Field variable expansion}

The steady-state pressure field $p^{a(\alpha)}(\mathbf{r})$ in the semi-unbounded acoustic domain $\Omega^{(\alpha)}$ is approximated by a solution expansion $\hat{p}^{a \alpha}(\mathbf{r})$ :

$$
p^{a(\alpha)}(\mathbf{r}) \simeq \hat{p}^{a(\alpha)}(\mathbf{r})=\sum_{w=1}^{n_{w}^{(\alpha)}} p_{w}^{(\alpha)} \Phi_{w}^{(\alpha)}(\mathbf{r})+\hat{p}_{q}^{(\alpha)}(\mathbf{r})=\Phi^{(\alpha)}(\mathbf{r}) \mathbf{p}_{w}^{(\alpha)}+\hat{p}_{q}^{(\alpha)}(\mathbf{r}) .
$$


The wave function contribution factors $p_{w}^{(\alpha)}$ are the weighting factors for each of the a priori selected wave functions $\Phi_{w}^{(\alpha)}(\mathbf{r})$. All weighting factors together form the vector of degrees of freedom $\mathbf{p}_{\mathbf{w}}^{(\alpha)}$. The corresponding defined wave functions are collected in the row vector $\boldsymbol{\Phi}^{(\alpha)}(\mathbf{r})$. The term $\hat{p}_{q}^{(\alpha)}(\mathbf{r})$ represents a particular solution resulting from source terms in the right hand side of the inhomogeneous Helmholtz equation (2).

The minimisation of boundary conditions (3) and (4)-(7) in the next step would involve the integration of residuals on infinitely large surfaces. In order to avoid these issues, the wave functions in the semi-unbounded acoustic domains are selected to fulfill the Helmholtz equation (2), the Sommerfeld radiation condition (3) and the BlochFloquet conditions (4)-(7 a priori), similarly as in the corresponding 2D case [44]. The wave functions $\Phi_{w}^{(\alpha)}(\mathbf{r})$ for a semi-unbounded periodic domain are based on a plane wave expansion:

$$
\Phi_{w}^{(\alpha)}(\mathbf{r}(x, y, z))=e^{-\mathrm{j}\left(k_{B F_{x w_{1}}}^{(\alpha)} x+k_{B F_{y w_{2}}}^{(\alpha)} y+k_{B F_{z w}}^{(\alpha)} z\right)} .
$$

The wave number components $k_{B F_{x w_{1}}}^{(\alpha)}$ and $k_{B F_{y w_{2}}}^{(\alpha)}$ are selected such that the periodicity conditions are fulfilled:

$$
\begin{aligned}
& k_{B F_{x w_{1}}}^{(\alpha)}=k_{a x}+\frac{2 w_{1}^{(\alpha)} \pi}{L_{x}^{(\alpha)}}, \\
& k_{B F_{y w_{2}}}^{(\alpha)}=k_{a y}+\frac{2 w_{2}^{(\alpha)} \pi}{L_{y}^{(\alpha)}},
\end{aligned}
$$

with $w_{1}^{(\alpha)}, w_{2}^{(\alpha)} \in \mathbb{Z}$. In order to fulfill the Helmholtz equation, the wave numbers $k_{B F_{z w}}^{(\alpha)}$ are selected as:

$$
k_{B F_{z w}}^{(\alpha)}= \pm \sqrt{k_{a}^{2}-\left(k_{B F_{x w_{1}}}^{(\alpha)}\right)^{2}-\left(k_{B F_{y w_{2}}}^{(\alpha)}\right)^{2}},
$$

and the sign of the root is selected to fulfill the Sommerfeld radiation condition ensuring that the waves are purely outgoing. For the two semi-unbounded acoustic domains $\Omega^{(1)}$ and $\Omega^{(2)}$, different signs are thus selected. Note that the subscript $w$ in Eq. (10) comprises all combinations of $w_{1}$ and $w_{2}$. In the following, the semi-infinite domain containing the incoming plane wave, is referred to as $\Omega^{(1)}$.

In order to satisfy the periodic nature of the problem, the particular solution $\hat{p}_{q}^{(\alpha)}(\mathbf{r})$ is required to be periodic or can be written as a superposition of periodic functions (e.g. plane wave(s)). In the considered model, an incoming plane wave is accounted for, using the following particular solution in Eq. (9):

$$
\hat{p}_{q}^{(\alpha)}(\mathbf{r})=A e^{-\mathbf{j} \mathbf{k}_{\mathbf{a}} \cdot \mathbf{r}},
$$

with $A$ the plane wave amplitude, $\mathbf{k}_{\mathbf{a}}=\left(k_{a x}, k_{a y}, k_{a z}\right)=\left(-k_{a} \sin \theta \cos \psi,-k_{a} \sin \theta \sin \psi,-k_{a} \cos \theta\right)$ the wave vector and $\theta$ and $\psi$ the propagation angles as defined in Fig. 1.

The definition of the wave function set, Eqs. (10)-(13) leads to an infinite amount of wave functions to be included. In practice, a linear, frequency-dependent truncation rule is suggested [67], such that all wave number components in the $x$ - and $y$-direction smaller than or equal to a truncation factor $N_{p}$ times the physical acoustic wave number $k_{a}$ are 
accounted for in the wave function set:

$$
\begin{aligned}
& w_{1, \text { max }}=\left\lceil\left(N_{p} k_{a}-k_{a x}\right) \frac{L_{x}}{2 \pi}\right\rceil, \\
& w_{2, \text { max }}=\left\lceil\left(N_{p} k_{a}-k_{a y}\right) \frac{L_{y}}{2 \pi}\right\rceil .
\end{aligned}
$$

Consequently, all wave numbers applying values of $w_{1}=\left[-w_{1, \max }, w_{1, \max }\right]$ en $w_{2}=\left[-w_{2, \max }, w_{2, \max }\right]$ are included in the numerical scheme. Note that this truncation rule leads to a stepped staircase-like behaviour when plotting the number of wave functions included versus frequency.

\subsubsection{Construction of the system of equations}

The wave functions (10) are selected to inherently fulfill the Helmholtz equation, the Sommerfeld radiation condition and the Bloch-Floquet boundary conditions inside the semi-unbounded acoustic domain. The wave function contribution factors should thus be determined such that the residuals on the interface with the FE part are minimised. These conditions depend on the physics involved in the FE part of the unit cell. The hybrid coupling is detailed later, in Section 3.2. In general, to construct the WB system matrices, a weighted residual approach is used, similar to FEM, expressing the weighting function in terms of the same wave functions to approximate the field variables. For an uncoupled WB problem a system of equations of the following form is obtained:

$$
\left[\mathbf{A}_{\mathbf{w w}}\right]\left\{\mathbf{p}_{\mathbf{w}}\right\}=\mathbf{f}_{\mathbf{w}}
$$

where $\mathbf{f}_{\mathbf{w}}$ results from non-zero boundary conditions and source contributions and $\mathbf{p}_{\mathbf{w}}$ collects the contribution factors of all subdomains $\alpha$.

\subsubsection{Solution and postprocessing}

The solution WB system of equations (17) leads to the contribution factors of each of the wave functions $\mathbf{p}_{\mathbf{w}}$. The back substitution of these contribution factors into the pressure expansions (9), using the wave functions defined in (10) and the plane wave definition (14) yields an analytical description of the approximated dynamic pressure field $\hat{p}^{a(\alpha)}(\mathbf{r})$. Also derived quantities, such as velocities and intensities can be obtained.

Due to the plane wave nature of the wave functions in the semi-unbounded periodic acoustic domains, the hemispherical reflection and transmission coefficients, $\mathcal{R}$ and $\mathcal{T}$, can be straightforwardly calculated based on the wave function contribution factors $p_{w}^{(\alpha)}$ of the semi-unbounded acoustic wave functions and the selected wave number components from Eq. (13):

$$
\begin{aligned}
& \mathcal{R}=\sum_{w} \frac{\mathfrak{R}\left(k_{B F_{z w}}^{(1)}\right)\left\|p_{w}^{(1)}\right\|^{2}}{k_{a z}\|A\|^{2}} \\
& \mathcal{T}=\sum_{w} \frac{\mathfrak{R}\left(k_{B F_{z w}}^{(2)}\right)\left\|p_{w}^{(2)}\right\|^{2}}{k_{a z}\|A\|^{2}}
\end{aligned}
$$


as evanescent waves do not carry energy. The absorption coefficient $\alpha$ cannot be straightforwardly evaluated using wave function contribution factors. It can, however, be evaluated using the conservation of energy as follows:

$$
\alpha=1-\mathcal{R}-\mathcal{T}
$$

Finally, the STL of the infinite periodic structure can be evaluated as follows:

$$
\mathrm{STL}=-10 \log _{10}(|\mathcal{T}|)
$$

\subsection{A hybrid WB-FE UC method for vibro-acoustic analysis}

This section of the paper treats the details of the hybrid coupling approach between the FEM and the WBM. A first subsection motivates the use of a hybrid approach, by comparing the properties of both subtechniques. Thereafter, the coupling framework is discussed in general.

\subsubsection{Comparison of FEM and WBM properties}

The FEM and the WBM both belong to the family of deterministic numerical modelling techniques. However, as they are based on two fundamentally different concepts (polynomial approximation functions in small elements versus analytically defined wave functions in large domains) they have different, yet very complementary properties:

- Field variable approximation: The FEM applies polynomial shape functions $\mathbf{N}(\mathbf{r})$, which interpolate local nodal values $\mathbf{v}_{\mathbf{f}}$ to describe the dynamic response variables. The WBM, applies the exact solutions of the underlying differential equations in the form of wave functions $\boldsymbol{\Phi}(\mathbf{r})$ which are weighted with contribution factors $\mathbf{p}_{\mathbf{w}}$.

- System matrix properties: The FEM modelling procedure typically leads to large, symmetric and sparsely populated system matrices. The WBM yields fully populated matrices with complex valued entries which are frequency dependent. For the same problem, the WBM matrices are generally much smaller than their FEM counterparts. Important to note is that, as with all Trefftz methods, the WBM suffers from ill-conditioned system matrices. This ill-conditioning becomes ever more severe as the number of approximation functions increases with frequency, and the wave function set exhibits more redundancy. Nonetheless, studies have shown that it is exactly this redundancy that provides stability [68]. As the WBM satisfies the discrete Picard condition [54], a meaningful solution can always be obtained. In this type of applications, ill-conditioning is rarely an issue due to the shape of the unit cell (rectangular prism) and the relatively low-frequency application; typically a limited number of wave functions are selected by the frequency dependent truncation rule.

- Geometrical flexibility: The inherent discretisation into small elements makes that the FEM has hardly any restrictions on the geometrical complexity of the considered problem domain. For the WBM, on the other hand, convergence is only ensured for convex geometries [54]. In other cases, the problem domain should be split up into convex subdomains and additional continuity conditions have to be enforced at the interface. Inclusions 
can be accounted for using the so-called multi-level framework [69]. For geometrically complex problems, in general, a strong increase in computational cost is observed and the efficiency of the WBM decreases.

- (Semi-) Infinite problems: Since the FEM is a domain discretisation technique, it is per definition not suited for (semi-) infinite problems. A number of solutions have been proposed, such as absorbing boundary conditions [47], infinite elements [48], and absorbing elements, such as the Perfectly Matched Layer (PML) [49]. All these approaches lead to an approximation of the Sommerfeld radiation condition (3) and besides, may increase the number of degrees of freedom and the calculation time or change the properties of the FE system of equations. In the WBM, infinite problems can be readily treated by ensuring that the used wave functions not only satisfy the governing differential equation(s), but also the (semi-) infinite radiation boundary conditions.

\subsubsection{Direct hybrid coupling framework}

As discussed in the introduction, nowadays, research is steered towards a variety of engineered metamaterials, which exploit localised details in the unit cell to obtain a system-wide behaviour. The geometrical flexibility of the FEM is thus an important feature. When studying their vibro-acoustic properties, however, typically transmission, absorption and reflection in a (semi-) infinite space are considered. Therefore, it is important to be able to treat infinite acoustic problems. These can be efficiently incorporated in the WBM. Both methods are thus very complimentary to tackle a part of the problem; their combination is an efficient best-of-two-worlds strategy.

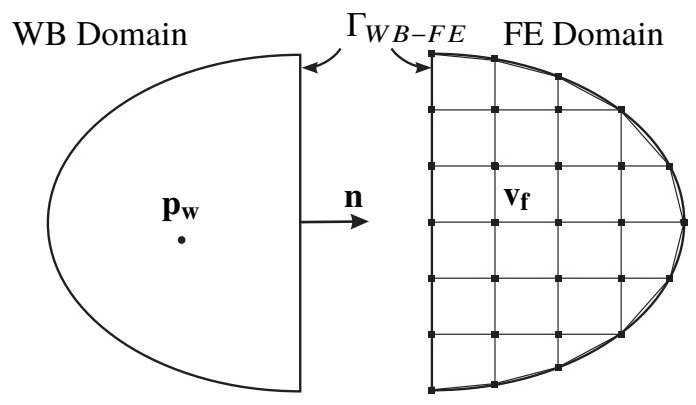

Figure 3: Direct hybrid FE-WBM coupling approach

Note that for clarity in the following the variables of the FE part are indicated with a subscript $f$ and these of the WB part with a subscript w. For ease of notation, the spatial r-dependency of all field variables, error residuals, shape functions, wave functions and particular solution terms is omitted in the remainder of the paper.

Using a direct coupling strategy (see Fig. 3), the mutual interactions between the FE and WB model can be directly introduced into the weighted residual formulations and thus into the uncoupled systems of equations. This direct strategy has the advantage that it does not introduce additional variables, and that the coupling terms are easy to interpret since they have a physical meaning. 
The exact way the coupling conditions are enforced is slightly different for each of the hybrid coupling types and depends on the primary model variables in the FE- and WB submodel. Depending whether the conditions are of the Dirichlet- (primary variables) or Neumann-type (derived variables) from the FE submodel viewpoint, the following logic is applied:

- Neumann conditions are always applied to the FE submodel. Because of the indirect nature of the WBM, enforcing boundary conditions always involves the minimisation of an error residual; it can never be enforced through direct variable substitution. Enforcing Neumann conditions using an error residual would require spatial derivatives of the polynomial shape functions of the FE submodel, which are less accurate. Therefore, Neumann coupling conditions are introduced into the FE submodel by a term of the form:

$$
\int_{\Gamma} \tilde{t}_{f} \mathcal{L}\left(\hat{p}_{w}^{a}\right) d \Gamma
$$

where $\tilde{t}_{f}$ are the FE submodel weighting functions and the operator $\mathcal{L}(\bullet)$ transforms the field variables of the WB model into the appropriate physical quantity that is required in the FE submodel's boundary residual. Typical examples can be found in Appendix A, e.g. the operator to calculate the normal acoustic velocity from the wave functions.

- Since Neumann conditions typically need to be enforced on the FE model, Dirichlet conditions are applied to the WB model. Again, due to the indirect nature of the WBM, enforcing the coupling conditions needs to be done throug minimisation of an error residual, and not through direct DOF elimination, as is conventionally done in the coupling of two FE models. This error residual is of the general form:

$$
\int_{\Gamma} \mathcal{N}\left(\tilde{t}_{w}\right)\left[\mathcal{V}\left(\hat{p}_{w}^{a}\right)-\hat{v}_{f}\right] \mathrm{d} \Gamma .
$$

In this condensed notation, the operator $\mathcal{V}(\bullet)$ transforms the indirect field variables of the WB model (i.e. the wave function weighting vector) into the direct DOFs of the FE model (i.e. the nodal field values). The operator $\mathcal{N}(\bullet)$ ensures that all residuals in the WB submodel are similarly scaled by writing them in energetic terms. Typical examples can be found in Appendix A, e.g. the acoustic-acoustic coupling where the pressure error residual is weighted with the acoustic particle velocity.

Adding these residuals (22)-(23) to the uncoupled residuals of the uncoupled FE and the WB models, respectively, and under the assumption that these combined weighted residual expressions should hold for any weighting function $\tilde{t}_{\bullet}$, the coupled equations can be written in terms of the unknown wave function contributions $\mathbf{p}_{\mathbf{w}}$ and the unknown nodal values $\mathbf{v}_{\mathbf{f}}$ :

$$
\left[\begin{array}{cc}
\mathbf{A}_{\mathbf{w w}}+\mathbf{C}_{\mathbf{w w}} & \mathbf{C}_{\mathbf{w f}} \\
\mathbf{C}_{\mathbf{f w}} & \mathbf{Z}_{\mathbf{f f}}+\mathbf{C}_{\mathbf{f f}}
\end{array}\right]\left[\begin{array}{c}
\mathbf{p}_{\mathbf{w}} \\
\mathbf{v}_{\mathbf{f}}
\end{array}\right]=\left[\begin{array}{c}
\mathbf{f}_{\mathbf{w}}+\mathbf{f}_{\mathbf{w w}} \\
\mathbf{f}_{\mathbf{f}}+\mathbf{f}_{\mathbf{f w}}
\end{array}\right],
$$

where the coupling between the FE- and WB submodel is ensured through the coupling terms $\mathbf{C}_{\mathbf{w w}}, \mathbf{C}_{\mathbf{w f}}, \mathbf{C}_{\mathbf{f w}}, \mathbf{C}_{\mathbf{f f}}, \mathbf{f}_{\mathbf{w w}}$ and $\mathbf{f}_{\mathbf{f w}}$. Note that for this application $\mathbf{A}_{\mathbf{w w}}$ is zero as the wave functions inherently satisfy the Sommerfeld radiation 
condition and the Bloch-Floquet periodicity boundary conditions; therefore, only interface residuals with the FE part have to be minimised. Further details on the derivation of these coupling terms for acoustic-acoustic, vibro-acoustic and poro-elastic-acoustic models can be found in Appendix A.

\subsubsection{Application of Bloch-Floquet boundary conditions}

On the WBM part, the periodicity conditions are explicitly accounted for in the definition of the wave functions. On the FE part of the unit cell, and consequently on the coupling matrices between FEM and WBM, Bloch-Floquet periodicity boundary conditions have to be applied. It is also assumed that the FE system matrices are not eliminated yet, i.e. without elimination of imposed DOFs or without eliminating DOFs due to the application of interface relations between adjacent $\mathrm{FE}$ subdomains. This allows to obtain the coupling matrices $\mathbf{C}_{\mathbf{w f}}$ and $\mathbf{C}_{\mathbf{f w}}$ directly as described in Section 3.2.2. The vector of all nodal values $\mathbf{v}_{\mathbf{f}}$ can be partitioned into 9 non-overlapping sets according to the DOFs on the boundary and the interior of the unit cell, as shown in Fig. 4:

$$
\mathbf{v}_{\mathbf{f}}=\left[\mathbf{v}_{\mathbf{B L}}^{\mathbf{T}} \mathbf{v}_{\mathbf{B R}}^{\mathbf{T}} \mathbf{v}_{\mathbf{T L}}^{\mathbf{T}} \mathbf{v}_{\mathbf{T R}}^{\mathbf{T}} \mathbf{v}_{\mathbf{L}}^{\mathbf{T}} \mathbf{v}_{\mathbf{R}}^{\mathbf{T}} \mathbf{v}_{\mathbf{B}}^{\mathbf{T}} \mathbf{v}_{\mathbf{T}}^{\mathbf{T}} \mathbf{v}_{\mathbf{I}}^{\mathbf{T}}\right]^{T},
$$

for which the nodes in the vector $\mathbf{v}_{\mathbf{i}}$, with $i \in\{B L, B R, T L, T R, L, R, B, T\}$ are located at the FE unit cell boundaries $\Gamma_{B F l}, \Gamma_{B F r}, \Gamma_{B F b}$ and $\Gamma_{B F t}$ at $x=0, x=L_{x}$ and $y=0, y=L_{y}$, while the nodes in the vector $\mathbf{v}_{\mathbf{I}}$ are located in the interior of the FE unit cell.

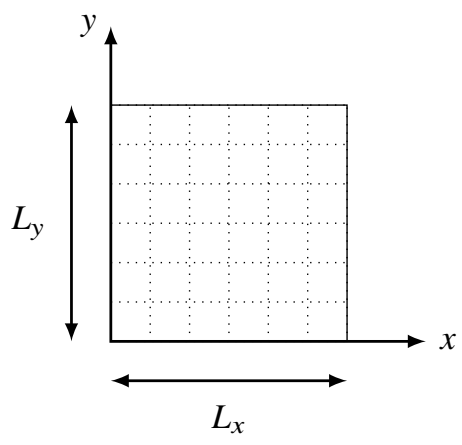

(a) Top view of the FE unit cell

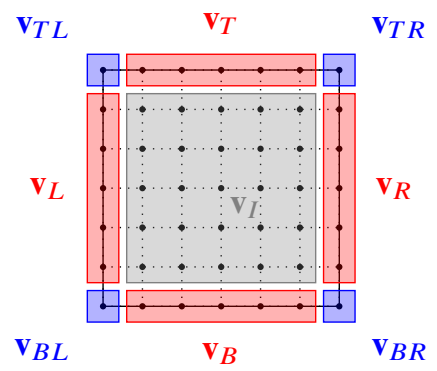

(b) FE model node groups

Figure 4: General rectangular unit cell of a 2D periodic structure

Application of the Bloch-Floquet periodicity conditions, Eq. (1), leads to the following relation, introducing the wave numbers $k_{a x}$ and $k_{a y}$ and the UC dimensions $L_{x}$ and $L_{y}$ imposed by the acoustic field and the geometrical 
periodicity:

$$
\mathbf{v}_{\mathbf{f}}=\mathbf{R}_{\mathbf{B F}}\left\{\begin{array}{c}
\mathbf{v}_{\mathbf{B L}} \\
\mathbf{v}_{\mathbf{L}} \\
\mathbf{v}_{\mathbf{B}} \\
\mathbf{v}_{\mathbf{I}}
\end{array}\right\}=\mathbf{R}_{\mathbf{B F}} \mathbf{v}_{\mathbf{B F}} ; \mathbf{R}_{\mathbf{B F}}=\left[\begin{array}{cccr}
\mathbf{I}_{\mathbf{B L}, \mathbf{B L}} & \mathbf{0} & \mathbf{0} & \mathbf{0} \\
e^{-j k_{a x} L_{x} \mathbf{I}_{\mathbf{B L}, \mathbf{B L}}} & \mathbf{0} & \mathbf{0} & \mathbf{0} \\
e^{-j k_{a y} L_{y} \mathbf{I}_{\mathbf{B L}, \mathbf{B L}}} & \mathbf{0} & \mathbf{0} & \mathbf{0} \\
e^{-j\left(k_{a_{x}} L_{x}+k_{a y} L_{y}\right)} \mathbf{I}_{\mathbf{B L}, \mathbf{B L}} & \mathbf{0} & \mathbf{0} & \mathbf{0} \\
\mathbf{0} & \mathbf{I}_{\mathbf{L}, \mathbf{L}} & \mathbf{0} & \mathbf{0} \\
\mathbf{0} & e^{-j k_{a x} L_{x} \mathbf{I}_{\mathbf{L}, \mathbf{L}}} & \mathbf{0} & \mathbf{0} \\
\mathbf{0} & \mathbf{0} & \mathbf{I}_{\mathbf{B}, \mathbf{B}} & \mathbf{0} \\
\mathbf{0} & \mathbf{0} & e^{-j k_{a y} L_{y} \mathbf{I}_{\mathbf{B}, \mathbf{B}}} & \mathbf{0} \\
\mathbf{0} & \mathbf{0} & \mathbf{0} & \mathbf{I}_{\mathbf{I}, \mathbf{I}}
\end{array}\right],
$$

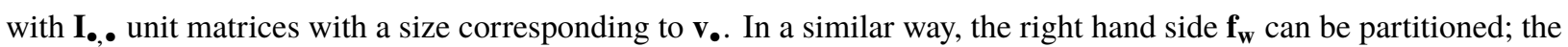
following relation is obtained under force equilibrium:

$$
\left\{\begin{array}{c}
\mathbf{f}_{\mathrm{BL}} \\
\mathbf{f}_{\mathbf{L}} \\
\mathbf{f}_{\mathrm{B}} \\
\mathbf{f}_{\mathbf{I}}
\end{array}\right\}=\mathbf{R}_{\mathrm{BF}}^{*} \mathbf{f}_{\mathbf{f}},
$$

where $\bullet *$ represents the Hermitian transpose of the matrix $\bullet$

Besides the periodicity conditions, the FE DOF elimination due to imposed Dirichlet conditions or due to interface continuity conditions, has to be accounted for. The elimination matrix $\mathbf{R}_{\text {FEM }}$ contains the relations between the retained DOFs $\mathbf{v}_{\mathbf{B F}}$, after elimination of the Bloch-Floquet conditions and the retained degrees of freedom $\mathbf{v}_{\mathbf{f}}^{\prime} \subseteq \mathbf{v}_{\mathbf{B F}}$ after a subsequent elimination due to all other imposed relations on the FE part:

$$
\mathbf{v}_{\mathbf{f}}=\mathbf{R}_{\mathrm{FEM}} \mathbf{v}_{\mathbf{B F}}=\mathbf{R}_{\mathrm{FEM}} \mathbf{R}_{\mathrm{BF}} \mathbf{v}_{\mathbf{f}}^{\prime}=\mathbf{R}_{\mathrm{red}} \mathbf{v}_{\mathbf{f}}^{\prime}
$$

Similarly, a relation for the retained nodal forces can be obtained:

$$
\mathbf{f}_{\mathbf{f}}=\mathbf{R}_{\text {red }}^{*} \mathbf{f}_{\mathbf{f}}^{\prime}
$$

By substituting expressions (28) and (29) into (24), the following eliminated system of equations is obtained:

$$
\left[\begin{array}{cc}
\mathbf{I} & \mathbf{0} \\
\mathbf{0} & \mathbf{R}_{\mathrm{red}}^{*}
\end{array}\right]\left[\begin{array}{cc}
\mathbf{A}_{\mathrm{ww}}+\mathbf{C}_{\mathrm{ww}} & \mathbf{C}_{\mathrm{wf}} \\
\mathbf{C}_{\mathrm{fw}} & \mathbf{Z}_{\mathrm{ff}}+\mathbf{C}_{\mathrm{ff}}
\end{array}\right]\left[\begin{array}{cc}
\mathbf{I} & \mathbf{0} \\
\mathbf{0} & \mathbf{R}_{\mathrm{red}}
\end{array}\right]\left[\begin{array}{c}
\mathbf{p}_{\mathrm{w}} \\
\mathbf{v}_{\mathbf{f}}^{\prime}
\end{array}\right]=\left[\begin{array}{c}
\mathbf{f}_{\mathrm{w}}+\mathbf{f}_{\mathrm{ww}} \\
\mathbf{R}_{\mathrm{red}}^{*} \mathbf{f}_{\mathbf{f}}+\mathbf{R}_{\mathrm{red}}^{*} \mathbf{f}_{\mathrm{fw}}
\end{array}\right],
$$

resulting in

$$
\left[\begin{array}{cc}
\mathbf{A}_{\mathbf{w w}}+\mathbf{C}_{\mathbf{w w}} & \mathbf{C}_{\mathrm{wf}}^{\prime} \\
\mathbf{C}_{\mathrm{fw}}^{\prime} & \mathbf{Z}_{\mathrm{ff}}^{\prime}+\mathbf{C}_{\mathrm{ff}}^{\prime}
\end{array}\right]\left[\begin{array}{c}
\mathbf{p}_{\mathrm{w}} \\
\mathbf{v}_{\mathbf{f}}^{\prime}
\end{array}\right]=\left[\begin{array}{c}
\mathbf{f}_{\mathrm{w}}+\mathbf{f}_{\mathrm{ww}} \\
\mathbf{f}_{\mathbf{f}}^{\prime}+\mathbf{f}_{\mathrm{fw}}^{\prime}
\end{array}\right],
$$




\subsubsection{Partitioned solution strategy}

As conventionally performed in hybrid WB-FE models [70], a matrix partitioning procedure based on the Schur complement $[71,72]$ is used for the solution in order to benefit from efficient solvers for sparse and dense matrix systems. In the following numerical examples, all sparse and dense matrices are solved using $A \backslash B$ in Matlab which selects the appropriate routine following [73]. After obtaining the DOFs in the WB and FE parts, standard postprocessing can be done in both domains and also the acoustic performance indicators, Eqs. (18)-(21), can be evaluated.

\section{Numerical validation cases}

This section applies the presented hybrid WB-FE UC method for three different problem cases. The first example considers a problem with an exact analytical solution available: a plane wave impinging on a rigidly backed poro-elastic multilayer system. This allows to validate the correctness of the hybrid implementation. The second example considers an absorption study of a thin porous layer with rigid inclusions of which results have been presented in literature. The third validation case studies the STL capability of a locally resonant metamaterial. All FE models presented in this section are obtained using Comsol Multiphysics 5.2a. The hybrid framework is implemented in Matlab R2016a.

\subsection{Poro-elastic multilayer configuration}

The first validation case considers a flat multi-layer system, consisting of a blanket, a screen and two foams discussed in Section 11.7.1 of [74]. The parameters of the multilayer are given in Table 1 and the applied air properties are listed in Table 2. The blanket, Blanket (1), is in contact with the surrounding air and the bottom foam layer, Foam (4), is bonded onto a rigid impervious wall. The Biot theory [62], together with the JCA model, is used to describe the dynamic behaviour within each of the four layers.

Table 1: Material properties of the multilayer poro-elastic validation case, taken from [74]

\begin{tabular}{lllllllllll}
\hline Material & Thickness $h$ & $\phi$ & $\sigma$ & $\alpha_{\infty}$ & $\Lambda$ & $\Lambda^{\prime}$ & $\rho_{1}$ & $E$ & $v$ & $\eta_{s}$ \\
& {$[\mathrm{~mm}]$} & {$[-]$} & {$\left[\mathrm{Ns} / \mathrm{m}^{4}\right]$} & {$[-]$} & {$[\mu \mathrm{m}]$} & {$[\mu \mathrm{m}]$} & {$\left[\mathrm{kg} / \mathrm{m}^{3}\right]$} & {$[\mathrm{Pa}]$} & {$[-]$} & {$[-]$} \\
\hline Blanket (1) & 4 & 0.98 & $34 \cdot 10^{3}$ & 1.18 & 60 & 86 & 41 & $286 \cdot 10^{3}$ & 0.3 & 0.015 \\
Screen (2) & 0.8 & 0.8 & $3.2 \cdot 10^{6}$ & 2.56 & 6 & 24 & 125 & $2.6 \cdot 10^{6}$ & 0.3 & 0.1 \\
Foam (3) & 5 & 0.97 & $87 \cdot 10^{3}$ & 2.52 & 36 & 118 & 31 & $143 \cdot 10^{6}$ & 0.3 & 0.055 \\
Foam (4) & 16 & 0.99 & $65 \cdot 10^{3}$ & 1.98 & 37 & 120 & 16 & $46.8 \cdot 10^{6}$ & 0.3 & 0.1 \\
\hline
\end{tabular}

As the multilayer system is of infinite extent, a UC with arbitrary dimension $L_{x}$ and $L_{y}$ can be chosen. Here, a $5 \mathrm{~cm}$ by $5 \mathrm{~cm}$ UC was selected; other dimensions have been verified and lead to similar results which are not reported here. Three different FE discretisation grids are considered as shown in Fig. 5. Linear and quadratic elements have been applied, respectively. The resulting number of elements and DOFs are summarised in Table 3. 


\begin{tabular}{ll}
\hline \multicolumn{2}{c}{ Air properties } \\
\hline$\rho_{a}\left[\mathrm{~kg} / \mathrm{m}^{3}\right]$ & 1.213 \\
$P_{0}[\mathrm{~Pa}]$ & $1.01325 \cdot 10^{5}$ \\
$\gamma[-]$ & 1.4 \\
$\eta[\mathrm{kg} /(\mathrm{ms})]$ & $1.839 \cdot 10^{-5}$ \\
\hline
\end{tabular}

Table 2: Air properties used within the multi-layer poro-elastic validation case.

Because of the symmetries, the response of the system is given by plane waves travelling in opposite directions. Therefore, it suffices to take into account the first wave numbers in Eqs. (11) and (12) applying $w_{1}=w_{2}=0$ to arrive at a correct representation of the dynamic field in the semi-infinite acoustic domain. This corresponds to a truncation factor of $N_{p}=1$ which ensures that all wave functions with wavelengths larger than or equal to the physical wavelength are included.

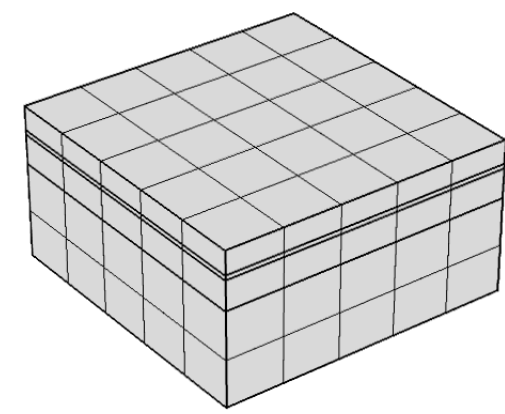

(a) FE multilayer mesh A

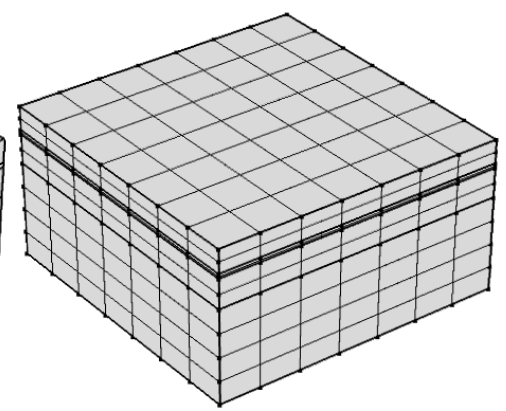

(b) FE multilayer mesh B

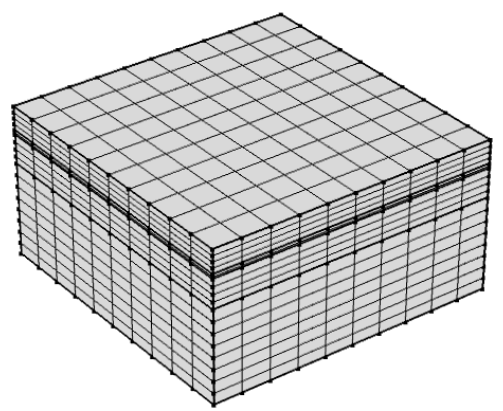

(c) FE multilayer mesh C

Figure 5: FE Unit cell models of the poro-elastic multilayer system

Table 3: Mesh information of the different FE meshes applied within the multi-layer poro-elastic validation case

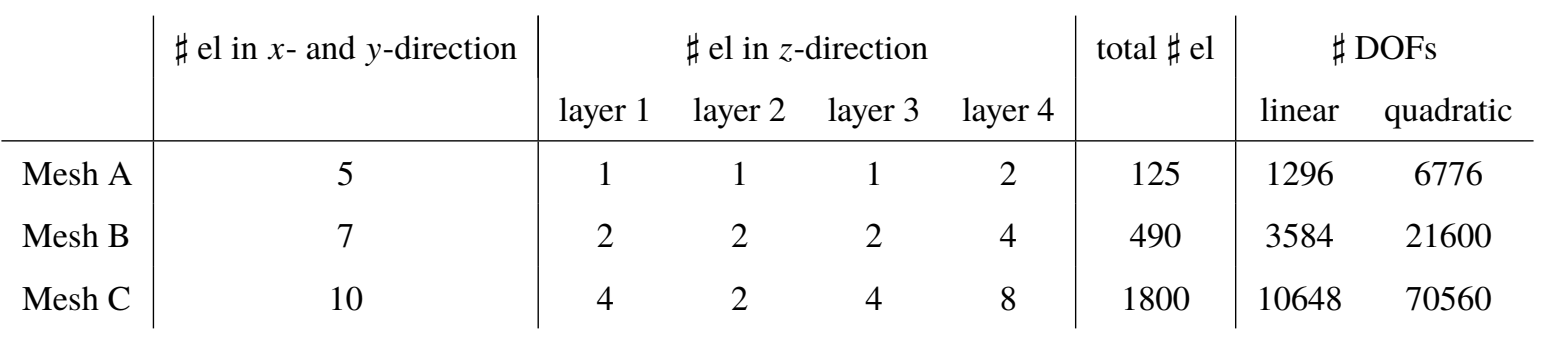

Three different angles of incidence are considered. The angle of elevation is varied, $\theta=0^{\circ}, 30^{\circ}, 45^{\circ}$ while keeping the azimuthal angle $\psi=0^{\circ}$ as it does not influence the results since the medium is isotropic in the plane, which has been validated as well. The absorption coefficient $\alpha$ of the multilayer system is predicted using the hybrid WB-FE UC method and the TMM [42], serving as a reference. The frequency band between 0 and $3000 \mathrm{~Hz}$ is considered, 
applying $10 \mathrm{~Hz}$ steps.

Figs. 6a, c and e show the obtained absorption coefficient for the three angles of incidence considered, using the TMM and the hybrid approach, applying the coarsest linear and the finest quadratic mesh in the FE part of the hybrid model. Fig. 6a corresponds to the result presented in [74]. The hybrid results, applying the most refined mesh lead to accurate results, whereas some deviations can be seen when the coarsest mesh, applying linear elements, is applied. To validate convergence, Figs. $6 \mathrm{~b}, \mathrm{~d}$ and $\mathrm{f}$ show the relative error between the hybrid results, considering all FE models presented in Table 3 and the TMM reference model, calculated as:

$$
\epsilon_{\bullet}=\left|\frac{\alpha_{\bullet}-\alpha_{T M M}}{\alpha_{T M M}}\right|,
$$

where $\alpha_{T M M}$ is the absorption coefficient predicted by the TMM and $\alpha_{\bullet}$ indicates the absorption coefficient obtained by any of the hybrid models. As indicated above, the WB submodel contains the two propagating waves that fully describe the mathematical solution; therefore only the FE discretisation can theoretically contribute to the prediction error. Indeed, by refining the FE mesh and/or increasing the order of elements, while keeping the WB truncation factor constant, more accurate results are obtained, as is to be expected. Moreover, the relative error increases when frequency increases, due to interpolation and pollution errors [75] in the FE part. When the response of the FE model becomes more complex, at oblique incidence around $600 \mathrm{~Hz}$, the coarsest mesh is insufficient to obtain engineering accuracy of $1 \%$. It should be noted, however, that for poro-elastic materials linear elements are rarely applied [76]. These figures validate the implementation of the hybrid model; for problems with a one-dimensional repsonse, the accuracy of the results is determined by the Finite Element part and care has to be taken to ensure that the mesh is sufficiently refined, as in standard FE analysis.

\subsection{Porous domain with rigid cylindrical inclusions}

The second validation case considers transmission through a porous unit cell with a rigid cylindrical inclusion. The porous medium is assumed to have a rigid frame and is modelled as an equivalent fluid medium, with a complex and frequency dependent effective density and effective bulk modulus, represented using the JCA model [74]. The porous material properties are listed in Table 4 and for the saturating air and the air in the acoustic domains, the properties from Table 2 are used. The thickness $L_{z}$ and the distance between the inclusions $L_{x}$ are set to $2 \mathrm{~cm}$. The inclusion is centered in the unit cell and has a radius $R$ of $7.5 \mathrm{~mm}$. A plane wave, incident under angle $\theta$, impinges on the structure.

The 2D equivalent, being a rigid circular inclusion was previously studied in $[44,77]$ and shown in Fig. 7. In order to validate the hybrid approach to the 2D reference results generated using the multi-level WBM [44], the azimuthal angle $\psi$ is kept to zero and the unit cell is extruded in the out-of-plane direction over a small, yet finite dimension $L_{y}=1 \mathrm{~mm}$. Note, however, that a more efficient hybrid model would be 2D, consisting of a 2D plane strain FE model coupled to a 2D WB model.

Three different meshes, named A, B and C, are considered, shown in Fig. 8, consisting of 3650, 13495 and 78050 DOFs respectively. In all models quadratic tetrahedral Lagrangian elements are applied. 


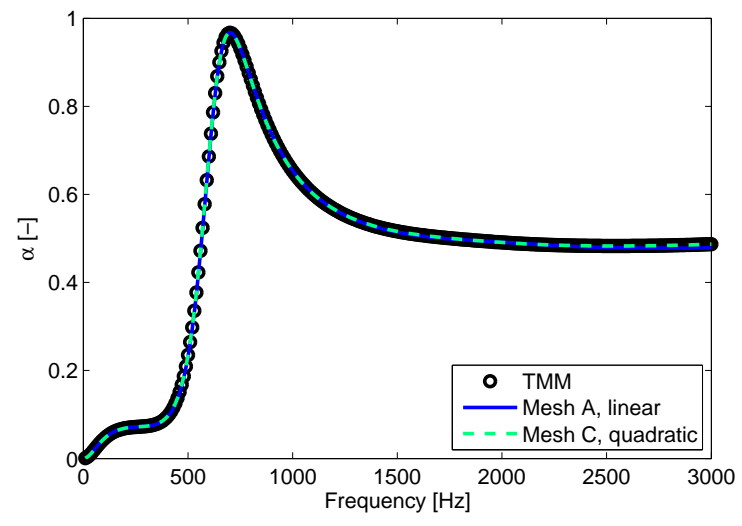

(a) Absorption coefficient, $\theta=0^{\circ}$

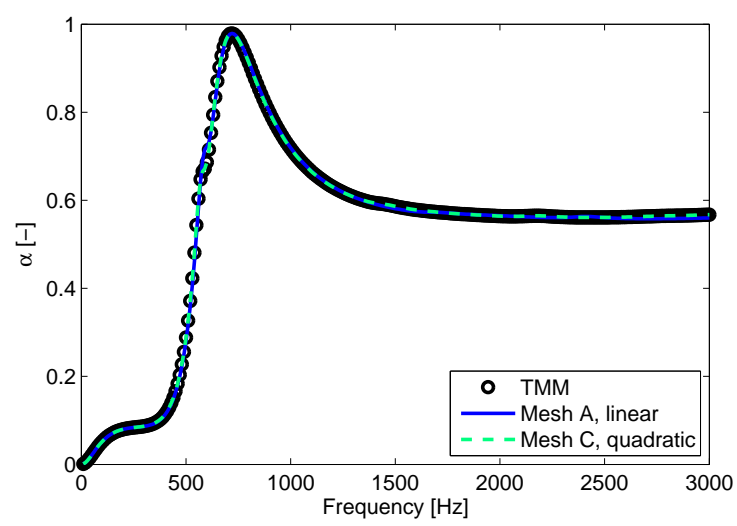

(c) Absorption coefficient, $\theta=30^{\circ}$

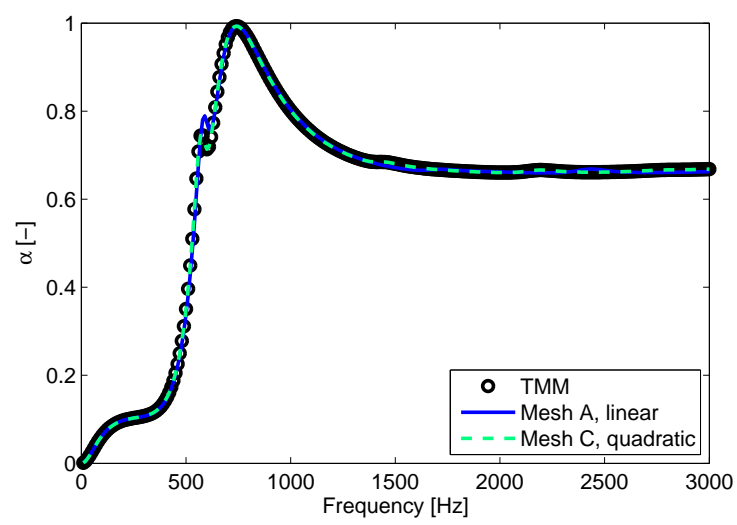

(e) Absorption coefficient, $\theta=45^{\circ}$

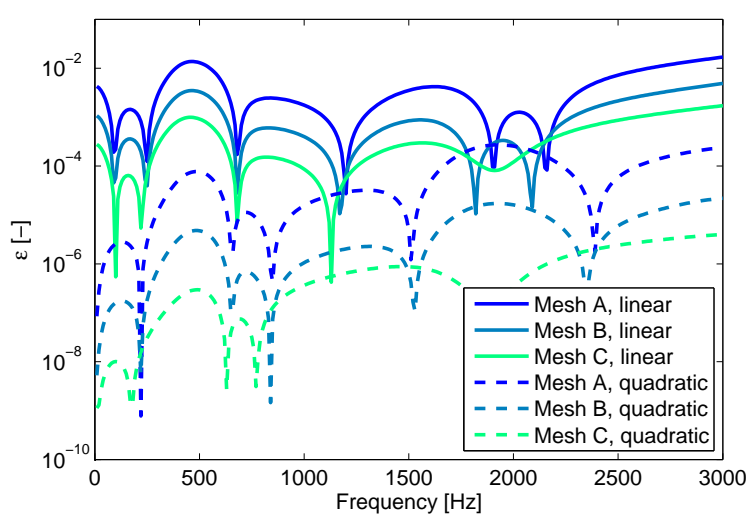

(b) Relative error, $\theta=0^{\circ}$

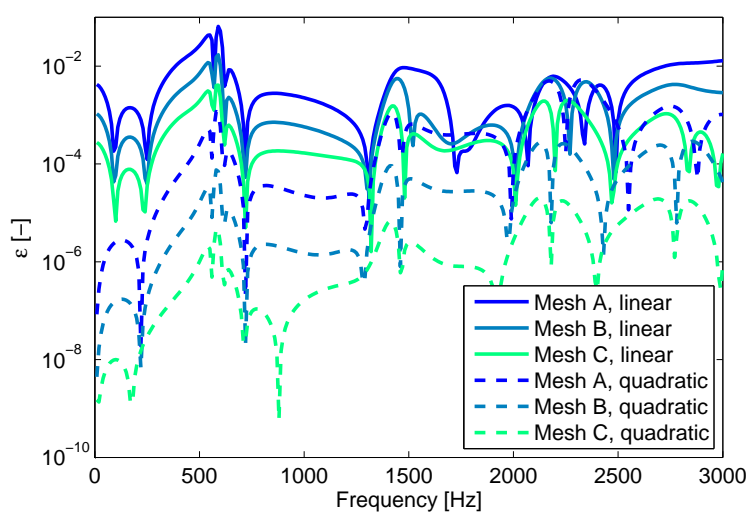

(d) Relative error, $\theta=30^{\circ}$

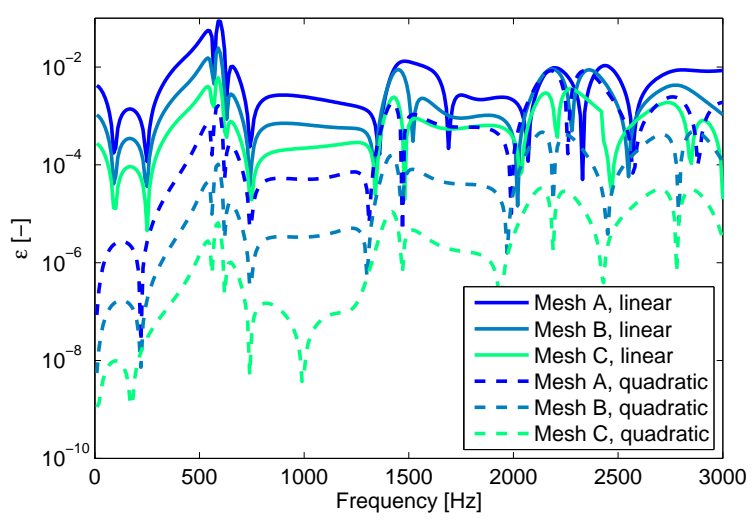

(f) Relative error, $\theta=45^{\circ}$

Figure 6: Absorption coefficient of the multilayer system as predicted by the TMM and the hybrid WB-FE UC method using the coarsest and the most refined mesh for three angles of incidence: $\theta=0^{\circ}(\mathrm{a}), \theta=30^{\circ}$ (b) and $\theta=45^{\circ}$ (c), and the corresponding relative error between the hybrid WB-FE UC method and the TMM results for the six different meshes, subfigures (b), (d), (f) 


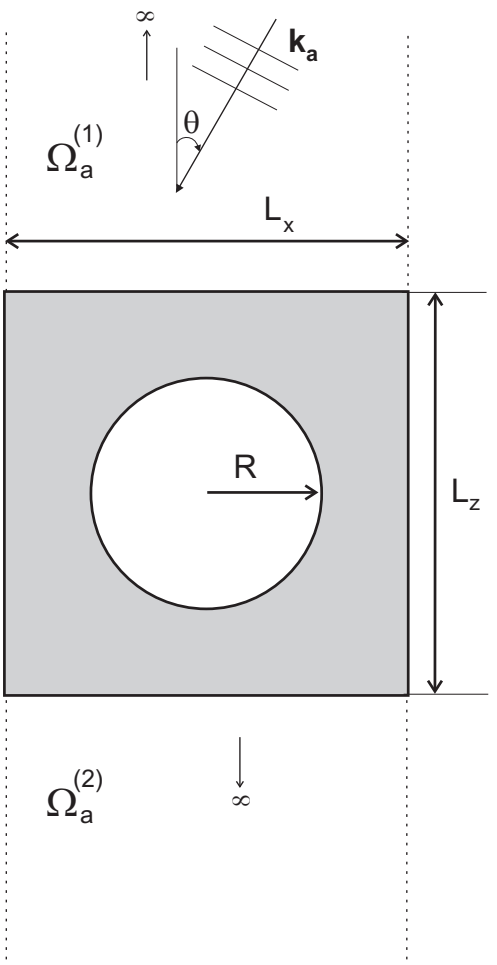

Figure 7: Problem setting of a porous domain with periodic rigid cylindrical inclusions

Table 4: Material properties of Fireflex foam [77].

\begin{tabular}{cccccc}
\hline Material & $\phi$ & $\sigma$ & $\alpha_{\infty}$ & $\Lambda$ & $\Lambda^{\prime}$ \\
& {$[-]$} & {$\left[\mathrm{Ns} / \mathrm{m}^{4}\right]$} & {$[-]$} & {$[\mu \mathrm{m}]$} & {$[\mu \mathrm{m}]$} \\
\hline Fireflex & 0.95 & $8.9 \cdot 10^{3}$ & 1.42 & 180 & 360 \\
\hline
\end{tabular}

The acoustic reflection, transmission and absorption coefficients have been calculated for the frequency range of $100 \mathrm{~Hz}$ to $18 \mathrm{kHz}$ and for two different angels of incidence $\theta=0^{\circ}, 30^{\circ}$ with different hybrid WB-FE UC models. Reference two-dimensional WB results are obtained, applying a truncation factor $N_{p}$ of 50 [44]. Note that a full WB calculation, also to model the structure with the inclusion, a high truncation factor is also required to accurately capture the dynamic behaviour within the unit cell. Nine different hybrid models are considered, combining each of the FE models described above with WB models using three different truncation factors: $N_{p}=1,2,3$.

Fig. 9 shows the reflection, transmission and absorption coefficient obtained for the two angles of incidence considered, using the Wave Based reference model (black circles) and the coarsest (solid blue line) and finest (dashed green line) hybrid model (i.e. using the coarsest/finest FE model combined with the lowest/highest WB truncation factor). For normal incidence perfect agreement is obtained for all models, whereas for oblique incidence the results of the coarsest model show a clear mismatch at higher frequencies.

The performance of the different models is further investigated by validating the convergence of the different 


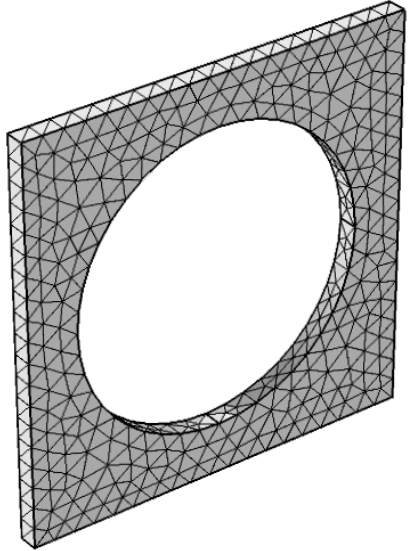

(a) FE UC model A

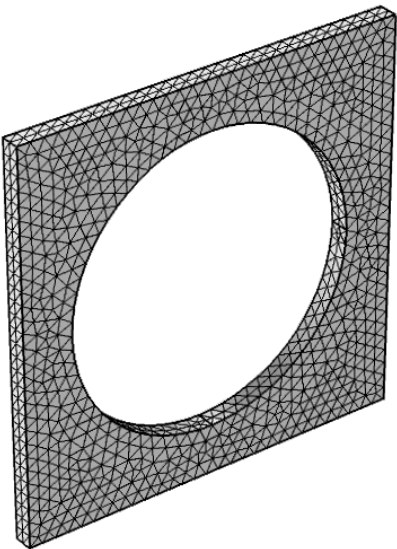

(b) FE UC model B

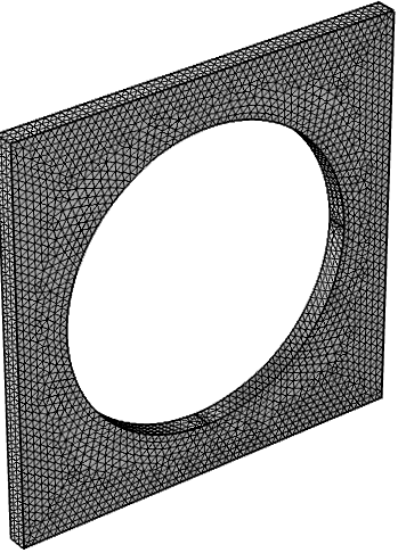

(c) FE UC model C

Figure 8: Unit cell models

models. Hybrid WB-FE models are known to have elbow-shaped convergence curves, see e.g. [78]. For a fixed frequency, when keeping the number of wave functions constant and decreasing the mesh size, accuracy increases until the WB part sets the final accuracy and the convergence curves stagnate. Further decreasing the mesh size does not lead to an additional gain in accuracy. On the other hand, for a fixed frequency, when keeping the number of FE DOFs constant and gradually increasing the number of wave functions included in the WB part, similar results are obtained: results converge, until the WB part is more accurate than the FE part which then sets the final accuracy.

Instead of running convergence simulations as described above, it is opted to investigate the performance of the different models directly over the full frequency range of interest by validating the relative error on the obtained absorption coefficient as follows:

$$
\epsilon_{\bullet}=\left|\frac{\alpha_{\bullet}-\alpha_{W B M}}{\alpha_{W B M}}\right|,
$$

where $\alpha_{W B M}$ is the absorption coefficient predicted by the two-dimensional WB reference model and $\alpha_{\bullet}$ indicates the absorption coefficient obtained by any of the hybrid models. These results mimic what an end user of the hybrid method would do: increasing the mesh size and/or increasing the truncation factor $N_{p}$. This representation has the advantage that is also reveals some peculiarities resulting from the WB truncation rule and the small dimensions of the unit cell applied.

Results for the nine hybrid models for normal incidence are shown in Fig. 10. Results associated to one particular FE mesh are shown in the same colour and results using the same WB truncation factor are shown using the same line style. In general, for all of the hybrid models, good accuracies are obtained, well below engineering accuracy of $1 \%$. At low frequencies, due to definition of the WB truncation rule, Eqs. (15)-(16), independently of the truncation factor set, the same number of wave functions are applied in each of the hybrid models. As a consequence, all hybrid models that apply the same mesh are coinciding. Small differences can be noticed for the three different FE meshes, but the 


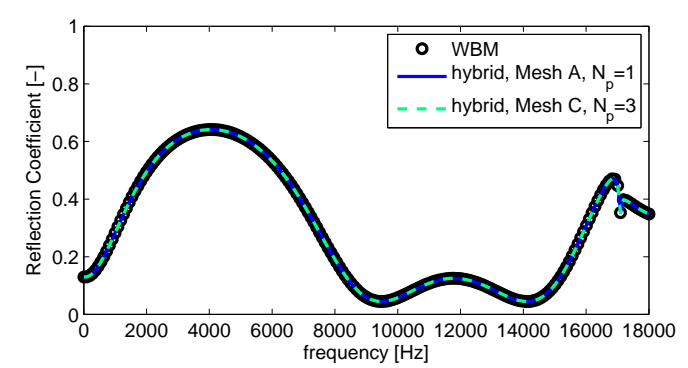

(a) Reflection coefficient, $\theta=0^{\circ}$

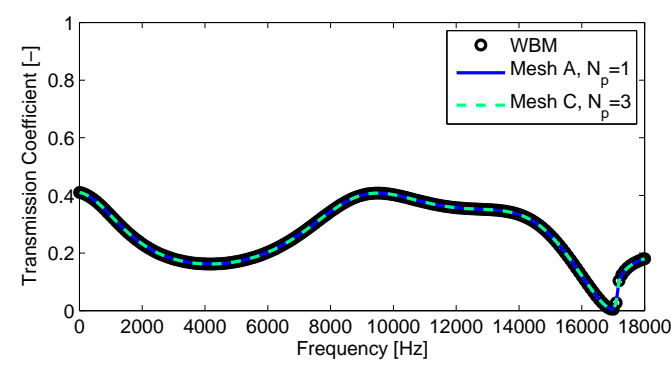

(c) Transmission coefficient, $\theta=0^{\circ}$

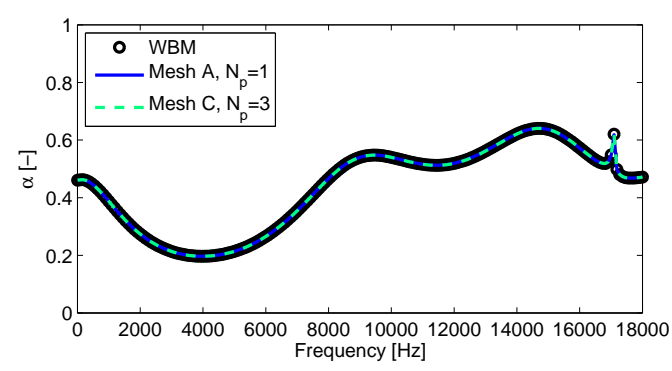

(e) Absorption coefficient, $\theta=0^{\circ}$

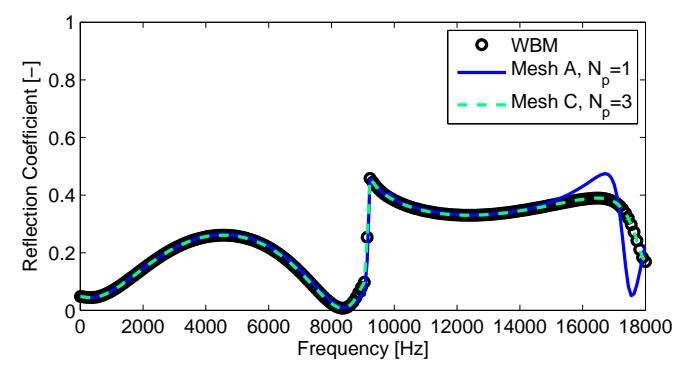

(b) Reflection coefficient, $\theta=30^{\circ}$

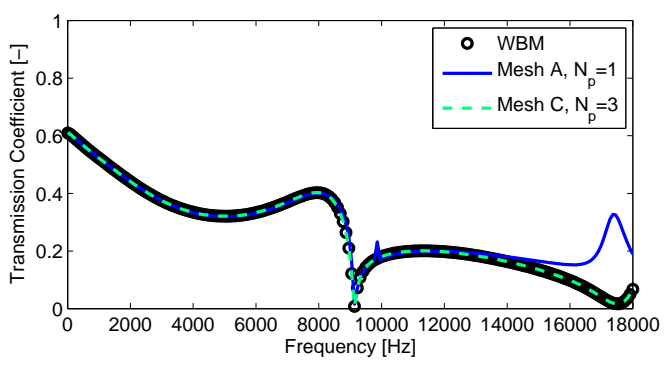

(d) Transmission coefficient, $\theta=30^{\circ}$

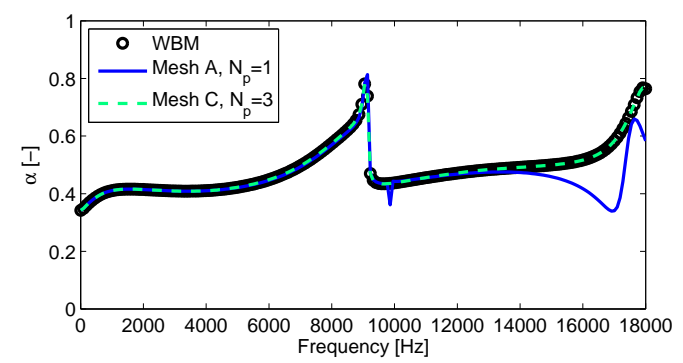

(f) Absorption coefficient, $\theta=30^{\circ}$

Figure 9: Reflection, Transmission and Absorption coefficient for $\theta=0^{\circ}$ (left column) and $\theta=30^{\circ}$ (right column) predicted by the two-dimensional WB reference model (black circles), the coarsest hybrid WB-FE UC model (blue solid line) and the most refined hybrid WB-FE UC model (green dashed line)

general accuracy is set by the WB part of the hybrid model. Around $5600 \mathrm{~Hz}$, the truncation factor $N_{p}=3$ leads to a sudden jump to a higher accuracy as a higher number of wave functions are accounted for in the WB part (dash-dotted lines). This is due to the definition of the truncation factor, Eqs. (15)-(16), which involves a ceiling-function. At this point, the accuracy of the applied FE model also plays a role and the results for the three models with different FE meshes are not on top of each other anymore. Note that for some frequencies the results for mesh C may be slightly less accurate than for mesh B, as the WB convergence is not necessarily monotonic. Of course, overall, the more accurate the FE mesh, the more accurate the end result. Around $8500 \mathrm{~Hz}$ the truncation factor $N_{p}=2$ leads to a jump in the number of wave functions accounted for in the WB expansion set. At that point, the hybrid models applying $N_{p}=2$ 


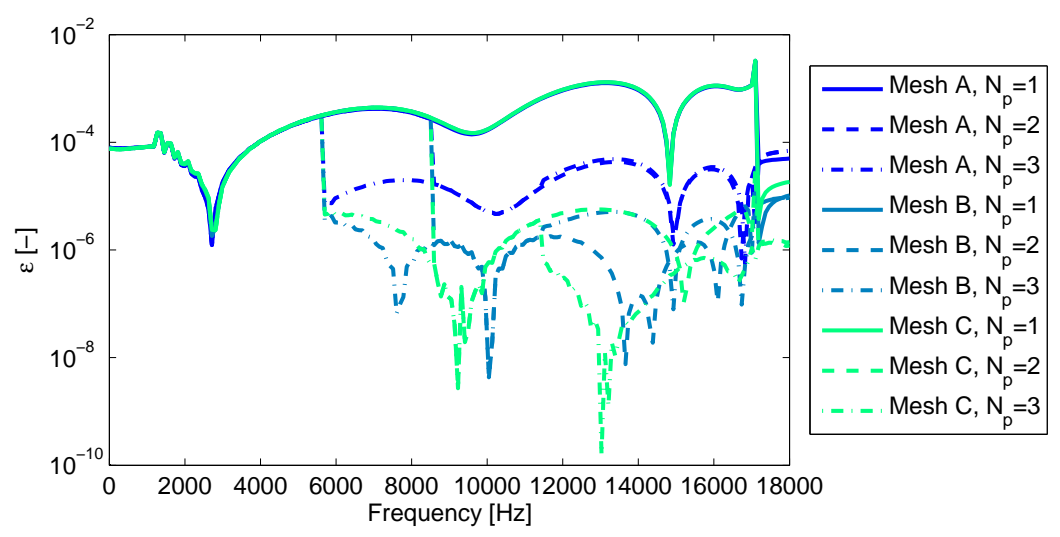

Figure 10: Relative error on the absorption coefficient for normal incidence of the periodic porous sample with rigid inclusions for the nine different hybrid models

and $N_{p}=3$ contain again an equal amount of wave functions and all dashed and dashed-dotted lines are coinciding again. Around $11600 \mathrm{~Hz}$, where the next jump in results take place, $N_{p}=3$ leads again to an increase in the number of wave functions leading to different accuracies for each of the three different meshes, comparing the curves for $N_{p}=2$ and $N_{p}=3$. Around $17000 \mathrm{~Hz}$, the number of wave function increases for $N_{p}=1$, again leading to an increase in accuracy. The accuracy of the results clearly converges when increasing the truncation factor and when increasing the number of FE elements, as expected.

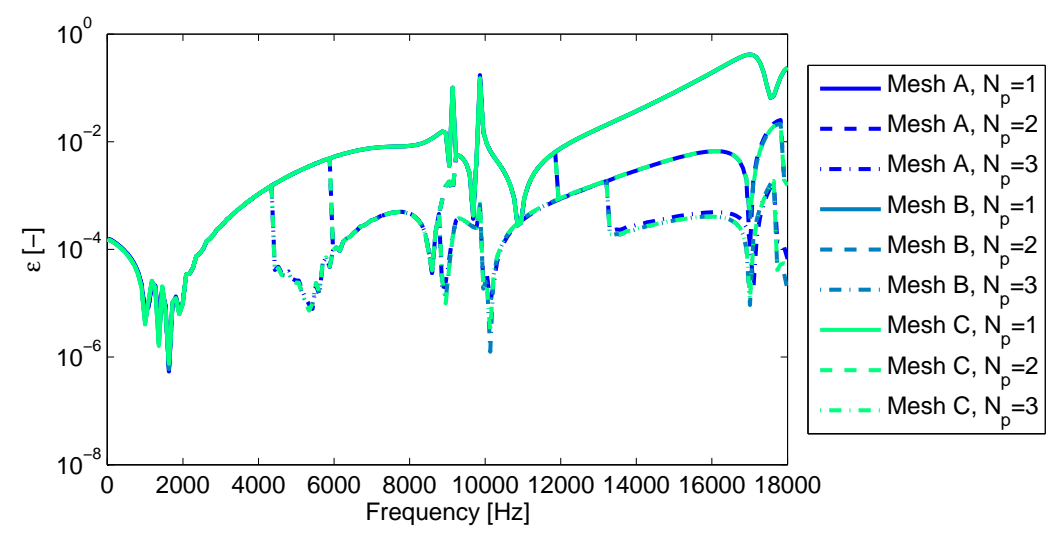

Figure 11: Relative error on the absorption coefficient for $\theta=30^{\circ}$ of the periodic porous sample with rigid inclusions for the nine different hybrid models

Fig. 11 shows similar results, now considering an angle of incidence $\theta=30^{\circ}$. The frequencies, where the truncation factors lead to an increase in the number of wave functions have shifted down in frequency, due to the higher value of $k_{a x}$ applied in Eq. (15). Trends are similar as for normal incidence, but the general accuracy is lower and it is mainly the WB part that is setting the accuracy, since results for different meshes but a constant truncation factor are 
coinciding, except at higher frequencies for $N_{p}=3$, where small differences can be observed due to the applied FE mesh. For most of the frequency band considered, the application of a truncation factor $N_{p}=2$ is sufficient to obtain engineering accuracy. A truncation factor $N_{p}=1$, which only accounts for waves larger than or about equal to the physical wave number, is insufficient, which is to be expected when dynamic response becomes more complex.

\subsection{Locally resonant metamaterial plate}

In this section, the vibro-acoustic performance of a locally resonant metamaterial is investigated by application of the hybrid FE-WB UC method and compared to the approach using dispersion curves adopted in [38].

The locally resonant metamaterial consists of a plate with periodically attached TVAs on a $5 \times 5 \mathrm{~cm}$ grid, to target the acoustically relevant out-of-plane flexural waves. The host structure is a $5 \mathrm{~mm}$ thick steel plate, with Young's modulus $E=210 \mathrm{GPa}$, Poisson coefficient $v=0.3$ and density $\rho=7800 \mathrm{~kg} / \mathrm{m}^{3}$, which has a coincidence frequency $f_{c}=2343 \mathrm{~Hz}$. The mass of the TVA is $20 \%$ of the plate mass, while its resonance frequency is tuned below coincidence at $1171 \mathrm{~Hz}$. The unit cell is modelled using 100 linear shell elements.

To assess the stop band behaviour, dispersion curves for the flexural waves are calculated following the inverse unit cell modelling approach described in [38]. For this study, wave propagation along the $x$-direction is considered, corresponding to an azimuthal propagation angle $\psi=0$ in the $x y$-plane. The dispersion curves for the flexural wave mode of the bare and metamaterial plate unit cell are shown in Figs. 12a and $12 \mathrm{~b}$ by means of black solid lines. Whereas no particular behaviour is present for the bare steel unit cell around the targeted frequency region, the metamaterial unit cell shows a clear band gap between 1154 and $1279 \mathrm{~Hz}$, since no freely propagating wave solutions are found in this frequency range. Therefore, this frequency range is expected to have increased vibro-acoustic attenuation.

As discussed in [38], the vibro-acoustic performance can be analysed through the dispersion curves by including the dispersion curve for the incident plane sound wave in air, $k_{a x}=-k_{a} \sin \theta$, along incidence elevation angle $\theta$, shown by means of dashed lines in Figs. 12a and 12b. By assessing the intersections between the structural and acoustic dispersion curves, coincidence frequencies for the corresponding incidence angles $\theta$ are identified. For the bare plate unit cell, efficiently radiating solutions are only found for frequencies higher than the coincidence frequency of $2343 \mathrm{~Hz}$, corresponding to grazing incidence condition $\theta=0^{\circ}$. For the metamaterial, however, the tuning of the band gap below this coincidence frequency causes a characteristic mode split for the targeted flexural wave type [38]. The modes before the stop band consists of in-phase motion between resonator and host structure, while after the stop band these modes are repeated at higher frequencies with out-of-phase motion. Since the repeated solutions after the band gap intersect with all acoustic solutions, an additional zone of efficiently radiating solutions is formed right after the stop band, below the original coincidence frequency, which can drastically influence the STL of the metamaterial plate.

However, based on this dispersion curve approach, no information is obtained about the magnitude of the STL increase due to the structural stop band and the impact of the additional coincidence zone. Therefore, the same bare and metamaterial unit cells are analyzed with the hybrid FE-WB UC method and the STL is calculated for the 


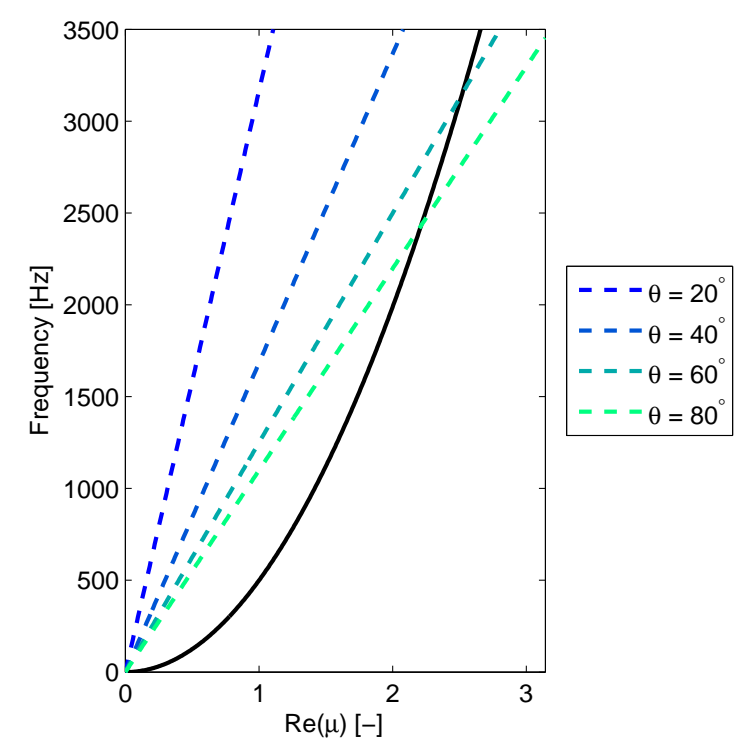

(a) Bare plate

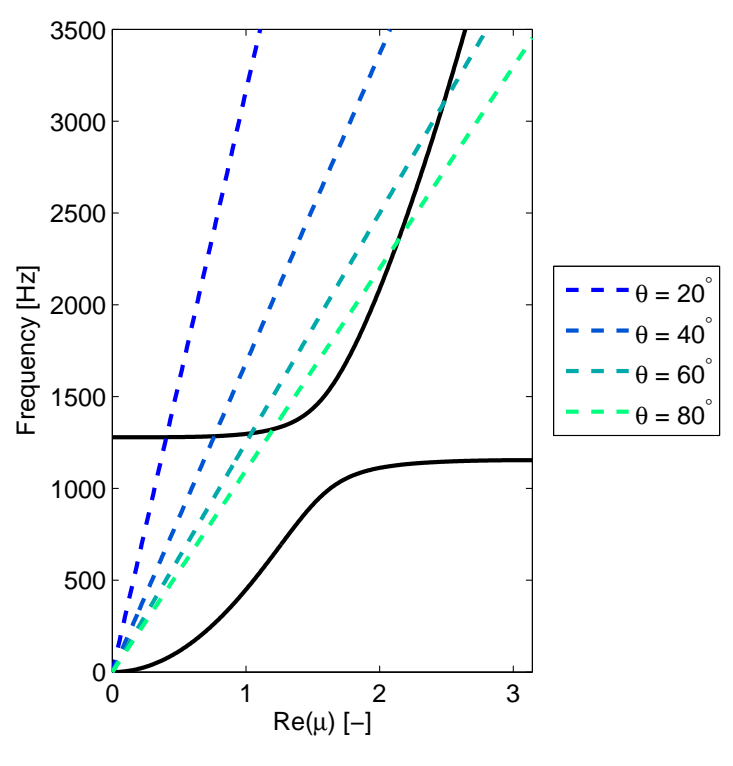

(b) Metamaterial plate

Figure 12: Dispersion curves $\left(\mu=k_{a x} L_{x}\right)$ along the $x$-direction for freely propagating structural waves (solid black lines) and dispersion curves of air for oblique plane wave incidence for incidence angles $\theta$ varying between $20^{\circ}$ and $80^{\circ}$ (dashed lines)

corresponding acoustic plane wave incidence angles $\psi=0^{\circ}$ and $\theta$ varying between $20^{\circ}$ and $80^{\circ}$ for the frequency range 1-3500 Hz with steps of $1 \mathrm{~Hz}$. Results are shown in Figs. 13a and 13b for the bare and metamaterial unit cell respectively.

For the bare unit cell, the incidence angles $\theta=80^{\circ}$ and $\theta=60^{\circ}$ show a STL dip at $2438 \mathrm{~Hz}$ and $3161 \mathrm{~Hz}$. These correspond to the intersections found between structural and acoustic dispersion curves in Fig. 12a. For the metamaterial, the STL in Fig. 13b shows a clear increase in the targeted frequency range around the tuned TVA frequency $[17,18,19]$. This zone of increased vibro-acoustic performance, is however followed by a zone of strongly reduced STL for all incidence angles. Strong STL dips are found at 1279, 1284, 1300 and $1323 \mathrm{~Hz}$ for acoustic plane wave incidence with $\theta$ varying from $20^{\circ}$ to $80^{\circ}$. These correspond to the additional intersections found in Fig. $12 \mathrm{~b}$ between the acoustic and structural dispersion curves right after the band gap, confirming the predicted additional low-frequency coincidence zone in the dispersion analysis. The STL dips at higher frequencies, $2357 \mathrm{~Hz}$ and $3108 \mathrm{~Hz}$ for $\theta=80^{\circ}$ and $\theta=60^{\circ}$, again correspond to the coincidence frequencies for classical flexural wave solutions, but now for the repeated flexural modes with out-of-plane motion between host structure and resonators, found by the intersections in Fig. 12b around the coincidence frequencies for the bare UC.

Both for the bare and metamaterial unit cell, the STL solutions resulting from the Hybrid FE-WB analysis are in good agreement with the predictions resulting from dispersion curve approach used in [38]. This examples highlights that the hybrid WB-FE UC method is an excellent tool for the analysis of novel emerging solutions. 


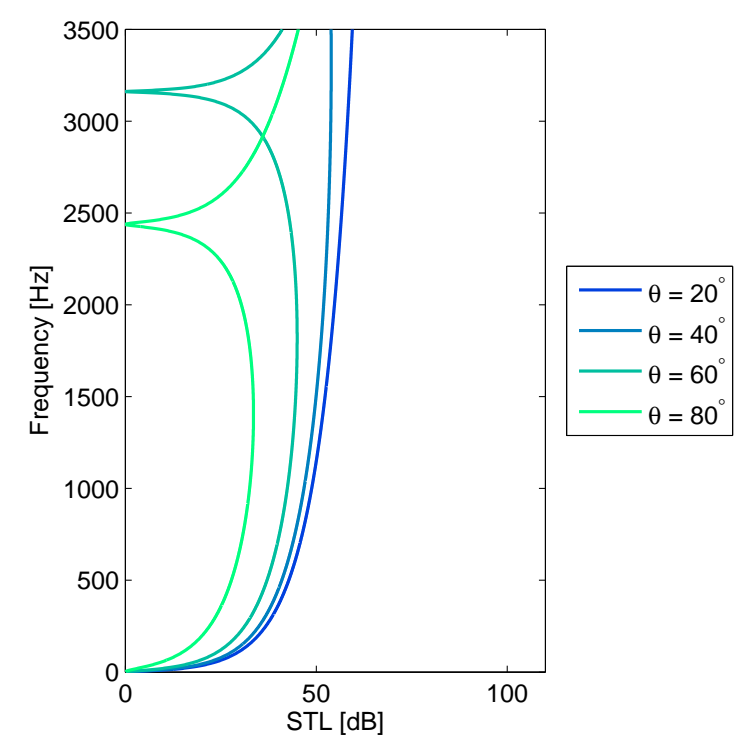

(a) Bare plate

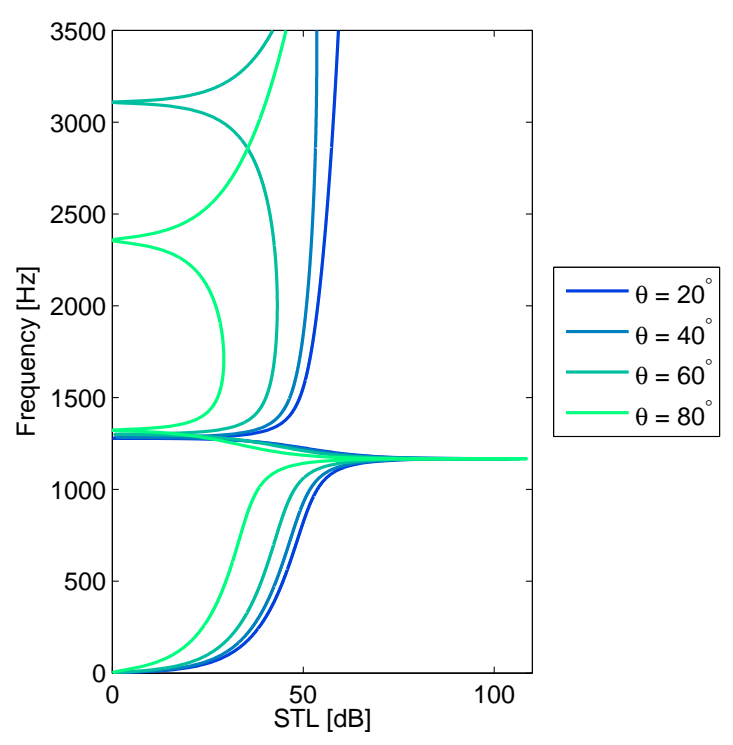

(b) Metamaterial plate

Figure 13: Sound transmission loss for acoustic plane wave incidence along the $x$-direction $\left(\phi=0^{\circ}\right)$ for incidence angles $\theta$ varying from $0^{\circ}$ to $80^{\circ}$

\section{Conclusions}

This paper presents a hybrid Wave Based - Finite Element unit cell method to predict acoustic performance indicators, such as the transmission, reflection and absorption coefficients, of in-plane periodic structures. The method combines the strengths of both WB and FE approaches, to meet the modelling requirements imposed by the ever more complex vibro-acoustic treatments emerging. The Finite Element method is used to model the unit cell and allows for geometrical details and complex physics to be included. The Wave Based Method is used to model the acoustic semiinfinite domains surrounding the planar periodic structure. A set of wave functions is presented that allows to directly account for the Sommerfeld radiation condition and the periodic boundary conditions, in order to avoid integrals to be evaluated on infinite surfaces or the calculation domain to be truncated leading to additional approximations. Both approaches are coupled in a direct sense, via a weighted integral formulation. A three-step procedure is used to solve the system of equations and to exploit the matrix properties of the subsystems: large, but sparse matrices for the FE part and small and fully populated matrices for the WB part. The hybrid WB-FE UC method is first validated for a problem which has an exact analytical solution. The method is also applied to a transmission problem with rigid circular inclusions, available in literature. By comparing the hybrid WB-FE UC results to the results of a refined WB reference model, its validity and accuracy are demonstrated. As a final example, a metamaterial plate is considered and the effects of stop bands and coincidence regions predicted by the dispersion curves of the structure and those of air, are verified in the sound transmission loss results. All examples illustrate this is a powerful tool to directly predict acoustic performance indicators of arbitrarily complex periodic structures. 


\section{Acknowledgements}

E. Deckers is a postdoctoral fellow of the Research Foundation - Flanders (F.W.O). The research of L. Van Belle is funded by a grant of the Research Foundation - Flanders (F.W.O). The EC is gratefully acknowledged for their support through the DEMETRA research project (GA324336). The Research Fund KU Leuven is gratefully acknowledged for its support. This research was partially supported by Flanders Make.

\section{Appendix A. Hybrid FE-WB coupling terms}

This section details the coupling terms for the different kinds of hybrid coupling between an acoustic WB model and an FE model describing acoustics, elastic- and poro-elastic materials, respectively. For ease of notation, the superscripts $\bullet(\alpha)$, indicating the WB subdomain number, have been omitted.

1. Acoustic-acoustic coupling - To couple two acoustic models, two conditions - one for each model - are necessary. These conditions are the force balance (pressure continuity) and the momentum balance (velocity perpendicular to the interface):

$$
\begin{array}{r}
p_{f e}^{a}-p_{w b}^{a}=0 \\
\mathcal{L}_{v_{n}}^{a}\left[p_{f e}^{a}\right]+\mathcal{L}_{v_{n}}^{a}\left[p_{w b}^{a}\right]=0
\end{array}
$$

where $p_{f e}^{a}$ and $p_{w b}^{a}$ represent the acoustic pressure approximation for the FE- and the WB submodel, respectively. The force balance in Eq. (A.1) is of the Dirichlet-type and is thus imposed on the WB subsystem by minimising the associated error residual on the interface.

$$
\int_{\Gamma_{w b}} \mathcal{L}_{v_{n}}^{a}\left[\tilde{p}_{w b}^{a}\right]\left[p_{f e}^{a}-p_{w b}^{a}\right] \mathrm{d} \Gamma=0
$$

Subsequently, the momentum continuity Eq. (A.2) is imposed on the FE model:

$$
\int_{\Gamma_{f e}} \tilde{p}_{f e}^{a} \mathcal{L}_{v_{n}}^{a}\left[p_{f e}^{a}\right] \mathrm{d} \Gamma=-\int_{\Gamma_{f e}} \tilde{p}_{f e}^{a} \mathcal{L}_{v_{n}}^{a}\left[p_{w b}^{a}\right] \mathrm{d} \Gamma .
$$

After substitution of the variable expansions, these two terms result in the following coupling matrices:

$$
\begin{aligned}
\mathbf{C}_{\mathbf{w w}}=\frac{j}{\omega \rho} \int_{\Gamma_{w b}} \frac{\partial \boldsymbol{\Phi}_{\mathbf{w}}^{\mathbf{T}}}{\partial \mathbf{n}} \boldsymbol{\Phi}_{\mathbf{w}} \mathrm{d} \Gamma & \mathbf{C}_{\mathbf{w f}}=-\frac{j}{\omega \rho} \int_{\Gamma_{w b}} \frac{\partial \boldsymbol{\Phi}_{\mathbf{w}}^{\mathbf{T}}}{\partial \mathbf{n}} \mathbf{N} \Gamma & \mathbf{f}_{\mathbf{w w}}=-\frac{j}{\omega \rho} \int_{\Gamma_{f e}} \frac{\partial \boldsymbol{\Phi}_{\mathbf{w}}^{\mathbf{T}}}{\partial \mathbf{n}} \hat{p}_{q} \mathrm{~d} \Gamma \\
\mathbf{C}_{\mathbf{f w}}=\mathbf{C}_{\mathbf{w f}}^{\mathbf{T}} & \mathbf{C}_{\mathbf{f f}}=\mathbf{0} & \mathbf{f}_{\mathbf{f w}}=\frac{j}{\omega \rho} \int_{\Gamma_{f e}} \mathbf{N}^{T} \frac{\partial \hat{p}_{q}}{\partial \mathbf{n}} \mathrm{d} \Gamma .
\end{aligned}
$$

Further details about the acoustic FE-WB coupling can be found in [70].

2. Vibro-acoustic coupling - To couple an acoustic and an elastic model, two conditions are necessary: one on the acoustic submodel and one in each direction for the elastic submodel. These interface conditions, describing 
force and momentum balance, can be expressed as follows:

$$
\begin{array}{r}
p_{w b}^{a}+\sigma_{f e, n}^{e}=0, \\
\sigma_{f e, s}^{e}=0, \\
\mathcal{L}_{v_{n}}^{a}\left[p_{w b}^{a}\right]-\mathrm{j} \omega u_{f e, n}^{e}(\mathbf{r})=0,
\end{array}
$$

where $u_{f e}^{e}$ represents the elastic displacement field, $\sigma_{f e}^{e}$ the elastic stress field and $p_{w b}^{a}$ the acoustic pressure in the WB submodel.

The force balance Eqs. (A.7)-(A.8) are both Neumann-type conditions and are thus elegantly introduced into the FE subsystem residual using the boundary residual.

$$
\begin{aligned}
& \int_{\Gamma_{f e}} \tilde{u}_{f e, n}^{e} \sigma_{f e, n}^{e} \mathrm{~d} \Gamma=-\int_{\Gamma_{f e}} \tilde{u}_{f e, n}^{e} p_{w b}^{a} \mathrm{~d} \Gamma, \\
& \int_{\Gamma_{f e}} \tilde{u}_{f e, s}^{e} \sigma_{f e, s}^{e} \mathrm{~d} \Gamma=0 .
\end{aligned}
$$

The momentum continuity Eq. (A.9) is introduced into the WB subsystem by minimising the error residual on the interface.

$$
\int_{\Gamma_{w b}} \tilde{p}_{w b}^{a}\left[\mathcal{L}_{v_{n}}^{a}\left[p_{w b}^{a}\right]-\mathrm{j} \omega u_{f e, n}^{e}\right] \mathrm{d} \Gamma=0
$$

After substitution of the variable expansions, these three terms result in the following coupling matrices:

$$
\begin{array}{rlrl}
\mathbf{C}_{\mathbf{w w}} & =\frac{1}{\omega^{2} \rho} \int_{\Gamma_{w b}} \boldsymbol{\Phi}_{\mathbf{w}}^{\mathbf{T}} \frac{\partial \boldsymbol{\Phi}_{\mathbf{w}}}{\partial \mathbf{n}} \mathrm{d} \Gamma & \mathbf{C}_{\mathbf{w f}}=-\int_{\Gamma_{w b}} \boldsymbol{\Phi}_{\mathbf{w}}^{\mathbf{T}} \mathbf{N} \mathrm{d} \Gamma & \mathbf{f}_{\mathbf{w w}}=-\frac{1}{\omega^{2} \rho} \int_{\Gamma_{w b}} \boldsymbol{\Phi}_{\mathbf{w}}^{\mathbf{T}} \frac{\partial \hat{\mathbf{p}}_{\mathbf{q}}}{\partial \mathbf{n}} \mathrm{d} \Gamma \\
\mathbf{C}_{\mathbf{f w}}=\mathbf{C}_{\mathbf{w f}}^{\mathbf{T}} & \mathbf{C}_{\mathbf{f f}}=\mathbf{0} & \mathbf{f}_{\mathbf{f w}}=\int_{\Gamma_{f e}} \mathbf{N}^{T} \hat{p}_{q} \mathrm{~d} \Gamma
\end{array}
$$

Further details about the vibro-acoustic FE-WB coupling can be found in [78].

3. Poro-elastic-acoustic (u,p) coupling - Coupling an acoustic and a poro-elastic model requires one condition for the acoustic model, one for the poro-elastic fluid phase, and one in each direction for the solid phase. These conditions describe the force- and momentum balance:

$$
\begin{array}{r}
\mathcal{L}_{v_{n}}^{a}\left[p_{w b}^{a}\right]-\mathrm{j} \omega u_{f e, n}^{t}=0, \\
\sigma_{f e, n}^{s}+(1-\phi) p_{w b}^{a}=0, \\
p_{f e}^{f}-p_{w b}^{a}=0, \\
\sigma_{f e, s}^{s}=0,
\end{array}
$$

where $u_{f e}^{t}$ represents the total poro-elastic displacement field, $\sigma_{f e}^{s}$ the stress field in the solid phase, $p_{f e}^{f}$ the pressure in the fluid phase an $\phi$ the porosity in the FE submodel and $p_{w b}^{a}$ the acoustic pressure in the WB submodel. 
After consecutive variable substitutions, which are further detailed in [79], the following residuals are obtained. The first two are of the Neumann-type and are thus applied to the boundary residual of the FE submodel:

$$
\begin{aligned}
\int_{\Gamma_{f e}} \tilde{u}_{f e, n}^{s} \sigma_{f e, n}^{s} \mathrm{~d} \Gamma & =-\int_{\Gamma_{f e}} \tilde{u}_{f e, n}^{s} p_{w b}^{a} \mathrm{~d} \Gamma \\
\int_{\Gamma_{f e}} \tilde{u}_{f e, s}^{s} \sigma_{f e, s}^{s} \mathrm{~d} \Gamma & =0 .
\end{aligned}
$$

The final two coupling terms are introduced into the FE subsystem and the WB subsystem, respectively, by minimising an error residual on the interface:

$$
\begin{gathered}
\int_{\Gamma_{f e}} \tilde{p}_{f e}^{f}\left[\frac{1}{\mathrm{j} \omega} \mathcal{L}_{v_{n}}^{a}\left[p_{w b}^{a}\right]-u_{f e, n}^{s}\right] \mathrm{d} \Gamma=0 \\
\int_{\Gamma_{w b}} \frac{1}{\mathrm{j} \omega} \mathcal{L}_{v_{n}}^{a}\left[\tilde{p}_{f e}^{a}\right]\left[p_{f e}^{f}-p_{w b}^{a}\right] \mathrm{d} \Gamma=0 .
\end{gathered}
$$

After substitution of the variable expansions, these four terms result in the following coupling matrices:

$$
\begin{aligned}
\mathbf{C}_{\mathbf{w w}}=\frac{1}{\omega^{2} \rho} \int_{\Gamma_{w b}} \frac{\partial \boldsymbol{\Phi}_{\mathbf{w}}^{\mathbf{T}}}{\partial \mathbf{n}} \boldsymbol{\Phi}_{\mathbf{w}} \mathrm{d} \Gamma & \mathbf{C}_{\mathbf{w f}}=-\frac{1}{\omega^{2} \rho} \int_{\Gamma_{w b}} \frac{\partial \boldsymbol{\Phi}_{\mathbf{w}}^{\mathbf{T}}}{\partial \mathbf{n}} \mathbf{N} \Gamma \quad \mathbf{f}_{\mathbf{w w}}=\frac{1}{\omega^{2} \rho} \int_{\Gamma_{w b}} \frac{\partial \boldsymbol{\Phi}_{\mathbf{w}}^{\mathbf{T}}}{\partial \mathbf{n}} \hat{p}_{q} \mathrm{~d} \Gamma \\
\mathbf{C}_{\mathbf{f w}}=\left[\begin{array}{c}
-\int_{\Gamma_{f e}} \mathbf{N}^{T} \boldsymbol{\Phi}_{\mathbf{w}} \mathrm{d} \Gamma \\
\frac{1}{\omega^{2} \rho} \int_{\Gamma_{f e}} \mathbf{N}^{T} \frac{\partial \boldsymbol{\Phi}_{\mathbf{w}}}{\partial \mathbf{n}} \mathrm{d} \Gamma
\end{array}\right] & \mathbf{C}_{\mathbf{f f}}=\left[\begin{array}{c}
\mathbf{0} \\
-\int_{\Gamma_{f e}} \mathbf{N}^{T} \mathbf{N d} \Gamma
\end{array}\right] \quad \mathbf{f}_{\mathbf{f w}}=\left[\begin{array}{c}
\int_{\Gamma_{f e}} \mathbf{N}^{T} \hat{p}_{q} \mathrm{~d} \Gamma \\
-\frac{1}{\omega^{2} \rho} \int_{\Gamma_{f e}} \mathbf{N}^{T} \frac{\partial \hat{p}_{q}}{\partial \mathbf{n}} \mathrm{d} \Gamma
\end{array}\right]
\end{aligned}
$$

Further details about the hybrid poro-elastic-acoustic FE-WB coupling can be found in [79].

\section{References}

[1] A. I. Taub, P. E. Krajewski, A. A. Luo, and J. N. Owens. The evolution of technology for materials processing over the last 50 years: The automotive example. The Journal of The Minerals, Metals \& Materials Society, 59(2):48-57, 2007.

[2] L. Goines and L. Hagler. Noise Pollution: A Modern Plague. Southern Medical Journal, 100:287-294, 2007.

[3] F. C. Sgard, X. Olny, N. Atalla, and F. Castel. On the use of perforations to improve the sound absorption of porous materials. Applied Acoustics, 66:625-651, 2005.

[4] J.-P. Groby, B. Brouard, O. Dazel, B. Nennig, and L. Kelders. Enhancing rigid frame porous layer absorption with three-dimensional periodic irregularities. The Journal of the Acoustical Society of America, 133:821-831, 2013.

[5] J.-P. Groby, C. Lagarrigue, B. Brouard, O. Dazel, and V. Tournat. Using simple shape three-dimensional rigid inclusions to enhance porous layer absorption. The Journal of the Acoustical Society of America, 136:1139-1148, 2014.

[6] T. Weisser, J.-P. Groby, O. Dazel, F. Gaultier, E. Deckers, S. Futatsugi, and L. Monteiro. Acoustic behavior of a rigidly backed poroelastic layer with periodic resonant inclusions by a multiple scattering approach. The Journal of the Acoustical Society of America, 139:617-629, 2016.

[7] C. Boutin and F.-X. Bécot. Theory and experiments on poro-acoustics with inner resonators. Wave Motion, 54:76-99, 2015.

[8] C. Lagarrigue, J.-P. Groby, V. Tournat, O. Dazel, and O. Umnova. Absorption of sound by porous layers with embedded periodic arrays of resonant inclusions. The Journal of the Acoustical Society of America, 134:4670-4680, 2013.

[9] J.-P. Groby, C. Lagarrigue, B. Brouard, O. Dazel, V. Tournat, and B. Nennig. Enhancing the absorption properties of acoustic porous plates by periodically embedding Helmholtz resonators. The Journal of the Acoustical Society of America, 137:273-280, 2015.

[10] C. Lagarrigue, J.-P. Groby, O. Dazel, and V. Tournat. Design of metaporous supercells by genetic algorithm for absoprtion optimization on a wide frequency band. Applied Acoustics, 102:49-54, 2016. 
[11] P. Sheng, X. X. Zhang, Z. Liu, and C. T. Chan. Locally resonant sonic materials. Physica B: Condensed Matter, 338(1):201-205, 2003.

[12] X. Zhou, X. Liu, and G. Hu. Elastic metamaterials with local resonances: An overview. Theoretical and Applied Mechanics Letters, 2(4):041001, 2012.

[13] M. I. Hussein, M. J. Leamy, and M. Ruzzene. Dynamics of phononic materials and structures: Historical origins, recent progress, and future outlook. Appl. Mech. Rev., 2014.

[14] C. Claeys, K. Vergote, P. Sas, and W. Desmet. On the potential of tuned resonators to obtain low-frequency vibrational stop bands in periodic panels. Journal of Sound and Vibration, 2013.

[15] C. Goffaux, J. Sánchez-Dehesa, A. Yeyati, P. Lambin, A. Khelif, J. Vasseur, and B. Djafari-Rouhani. Evidence of Fano-like interference phenomena in locally resonant materials. Physical Review Letters, 88:225502, 2002.

[16] C. Claeys, E. Deckers, B. Pluymers, and W. Desmet. A lightweight vibro-acoustic metamaterial demonstrator: Numerical and experimental investigation. Mechanical Systems and Signal Processing, 2016.

[17] Y. Xiao, J. Wen, and X. Wen. Sound transmission loss of metamaterial-based thin plates with multiple subwavelength arrays of attached resonators. Journal of Sound and Vibration, 2012.

[18] H. Zhang, J. Wen, Y. Xiao, G. Wang, and X. Wen. Sound transmission loss of metamaterial thin plates with periodic subwavelength arrays of shunted piezoelectric patches. 343, 2015.

[19] Y. Song, L. Feng, J. Wen, D. Yu, and X. Wen. Reduction of the sound transmission of a periodic sandwich plate using the stop band concept. Composite Structures, 128:428-436, 2015.

[20] Z. Yang, H. M. Dai, N. H. Chan, G. C. Ma, and P. Sheng. Acoustic metamaterial panels for sound attenuation in the 50-1000 Hz regime. Applied Physics Letters, 96:041906, 2010.

[21] J. Mei, G. Ma, M. Yang, Z. Yang, W. Wen, and P. Sheng. Dark acoustic metamaterials as super absorbers for low-frequency sound. Nature Communications, 3:756, 2012.

[22] X. Hu, C. Chan, and J. Zi. Two-dimensional sonic crystals with Helmholtz resonators. Physical Review E, 71:055601, 2005.

[23] D. Elford, L. Chalmers, F. Kusmartsev, and G. Swallowe. Matryoshka locally resonant sonic crystal. The Journal of the Acoustical Society of America, 130:2746-2755, 2011.

[24] M. Miniaci, A. Krushynska, A. B. Movchan, F. Bosia', and N. M. Pugno'. Spider web-inspired acoustic metamaterials. Applied Physical Letters, 109:071905, 2016.

[25] Y. Li, B. Liang, Z. Ming, X. Ye Zou, and X. Zhao. Reflected wavefront manipulation based on ultrathin planar acoustic metasurfaces. Scientific Reports, 3:02546, 2013

[26] G. Ma, M. Yang, S. Xiao, and P. Sheng. Acoustic metasurface with hybrid resonances. Nature Materials, 13:873-878, 2015.

[27] L. Schwan, O. Umnova, and C. Boutin. Sound absorption and reflection from a resonant metasurface: Homogenisation model with experimental validation. Wave Motion, 72:154-172, 2017.

[28] S. W. Ren, H. Meng, F. X. Xin, and T. J. Lu. Ultrathin multi-slit metamaterial as excellent sound absorber: Influence of micro-structure. Journal of Applied Physics, 119:014901, 2016.

[29] J. Yang, J. S. Lee, and Y. Y. Kim. Metaporous layer to overcome the thickness constraint for broadband sound absorption. Journal of Applied Physics, 117:174903, 2015.

[30] H. Ruiz, C. Claeys, E. Deckers, and W. Desmet. Numerical and experimental study of the effect of microslits on the normal absorption of structural metamaterials. Mechanical Systems and Signal Processing, 70:904-918, 2016.

[31] J.-P. Groby, W. Huang, A. Lardeau, and Y. Aurégan. The use of slow sound to design simple sound absorbing materials. Journal of Applied Physics, 117:124903, 2015.

[32] J.-P. Groby, R. Pommier, and Y. Aurégan. Use of slow sound to design perfect and broadband passive sound absorbing materials. The Journal of the Acoustical Society of America, 139:16601671, 2016.

[33] N. Jiménez, W. Huang, V. Romero-García, V. Pagneux, and J.-P. Groby. Ultra-thin metamaterial for perfect and quasi-omnidirectional sound absorption. Applied Physics Letters, 109:121902, 2016. 
[34] J. Yang, J. S. Lee, and Y. Y. Kim. Multiple slow waves in metaporous layers for broadband sound absorption. Journal of Physics D Applied Physics, 50:015301, 2017.

[35] F. Setaki, M. Tenpierik, M. Turin, and A. van Timmeren. Acoustic absorbers by additive manufacturing. Building and Environment, 72:188-200, 2014.

[36] F. Bloch. Über die Quantenmechanik der Elektronen in Kristallgittern. Zeitschrift für Physik A Hadrons and Nuclei, 52:555-600, 1929.

[37] L. Brillouin. Wave propagation in periodic structures. McGraw-Hill Book Company, 2nd edition, 1946.

[38] C. Claeys, P. Sas, and W. Desmet. On the acoustic radiation efficiency of local resonance based stop band materials. Journal of Sound and Vibration, 333:3203-3213, 2014.

[39] D. J. Mead. Plates with regular stiffening in acoustic media: vibration and radiation. The Journal of the Acoustical Society of America, 88(1):391-401, 1990.

[40] P. Li, S. Yao, and X. Zhou. effective medium theory of thin-plate acoustic metamaterials. The Journal of the Acoustical Society of America, 135:1844-1852, 2014.

[41] M. Oudich, X. Zhou, and M. Badreddine Assouar. General analytical approach for sound transmission loss analysis through a thick metamaterial plate. Journal of Applied Physics, 116(19):193509, 2014.

[42] B. Brouard, D. Lafarge, and J. F. Allard. A general method of modeling sound propagation in layered media. Journal of Sound and Vibration, 183:129-142, 1995.

[43] A. Parrinello and G. L. Ghiringhelli. Transfer matrix representation for periodic planar media. Journal of Sound and Vibration, $371: 196$ $209,2016$.

[44] E. Deckers, C. Claeys, O. Atak, J.-P. Groby, O. Dazel, and W. Desmet. A wave based method to predict the absorption, reflection and transmission coefficent of two-dimensional rigid frame porous structures with periodic inclusions. Journal of Computational Physics, 312:115-138, 2016

[45] J.-P. Groby, A. Wirgin, and E. Ogam. Acoustic response of a periodic distribution of macroscopic inclusions within a rigid frame porous plate. Waves in Random and Complex Media, 18:409-433, 2008

[46] R. R. Cook, D. S. Malkus, M. E. Pelsha, and R. J. Witt. Concepts and Applications of Finite Element Analysis. Wiley, Madison, 2002.

[47] D. Givoli. High-order local non-reflecting boundary conditions: A review. Wave Motion, 39:319-326, 2004.

[48] P. Bettess. Infinite elements. Penshaw Press, 1992.

[49] J.-P. Berenger. A Perfectly Matched Layer for the Absoprtion of Electromagnetic Waves. Journal of Computational Physics, 114:185-200, 1994.

[50] E. Bécache, P. Joly, and C. Tsogka. Fictitious domains, mixed finite elements and perfectly matched layers for $2 \mathrm{D}$ elastic wave propagation. J. Comp. Acous., 9(3):1175-1203, 2001.

[51] A. Bermúdez, L. Hervella-Nieto, A. Prieto, and R. Rodríguez. Perfectly matched layers for time-harmonic second order elliptic problems. Archives of Computational Methods in Engineering, 17:77-107, 2010.

[52] B. Assouar, M. Oudich, and X. Zhou. Acoustic metamaterials for sound mitigation. Comptes Rendus Physique, 17, 2016.

[53] S.G. Johnson. Notes on perfectly matched layers, http://www-math.mit.edu/ stevenj/18.369/spring09/pml.pdf.

[54] W. Desmet. A wave based prediction technique for coupled vibro-acoustic analysis. KU Leuven, division PMA, PhD. thesis 98D12, 1998.

[55] E. Deckers, O. Atak, L. Coox, R. D’Amico, H. Devriendt, S. Jonckheere, K. Koo, B. Pluymers, D. Vandepitte, and W. Desmet. The Wave Based Method: an overview of 15 years of research. Wave Motion, 51:550-565, 2014

[56] E. Trefftz. Ein Gegenstück zum Ritzschen Verfahren. In Proceedings of the $2^{\text {nd }}$ International Congress on Applied Mechanics, Zurich, Switzerland, pages 131-137, 1926.

[57] M. Collet, M. Ouisse, M. Ruzzene, and M. N. Ichchou. Floquet-Bloch decomposition for the computation of dispersion of two-dimensional periodic, damped mechanical systems. International Journal of Solids and Structures, 48:2837-2848, 2011.

[58] B. Mace and E. Manconi. Modelling wave propagation in two-dimensional structures using finite element analysis. Journal of Sound and Vibration, 318:3169-3180, 2008. 
[59] L. D. Landau and E. M. Lifshitz. Theory of Elasticity. Pergamon Press, 1975.

[60] E. Reissner. The effect of transverse shear deformation on the bending of elastic plates. Journal of Applied Mechanics, 12:69-77, 1945.

[61] R. D. Mindlin. Influence of rotary inertia and shear on flexural motions of isotropic, elastic plates. Journal of Applied Mechanics, 18:31-38, 1951.

[62] M. A. Biot. The theory of propagation of elastic waves in a fluid-saturated porous solid. I. Low frequency range. II. Higher frequency range. The Journal of the Acoustical Society of America, 28:168-191, 1956.

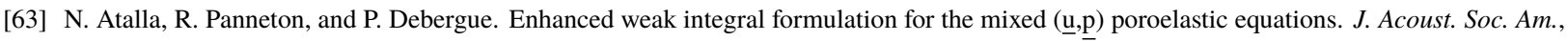
109:3065-3068, 2001.

[64] S. Jonckheere. Wave Based and Hybrid Methodologies for Vibro-acoustic Simulation with Complex Damping Treatments. KU Leuven, PhD. thesis, 2015.

[65] O.C. Zienkiewicz and R. L. Taylor. The finite element method, volume 3. McGraw-hill London, 1977.

[66] D. Colton and R. Kress. Inverse acoustic and electromagnetic scattering theory. Springer-Verlag, Berlin, Heidelbert, New York, 2nd edition, 1998.

[67] B. Van Genechten, O. Atak, B. Bergen, E. Deckers, S. Jonckheere, J.S. Lee, A. Maressa, K. Vergote, B. Pluymers, D. Vandepitte, and W. Desmet. An efficient Wave Based Method for solving Helmholtz problems in three-dimensional bounded domains. Engineering Analysis with Boundary Elements, 36:63-75, 2012.

[68] Daan Huybrechs. On the benefits of ill-conditioning in Trefftz-type methods and other non-polynomial discretisation of wave problems. In Innovations in Wave Modelling 2017, July 2017.

[69] B. Van Genechten, K. Vergote, D. Vandepitte, and W. Desmet. A Multi-Level Wave Based numerical modelling framework for the steady-state dynamic analysis of bounded Helmholtz problems with multiple inclusions. Computer Methods in Applied Mechanics and Engineering, 199:1881-1905, 2010.

[70] B. van Hal, W. Desmet, D. Vandepitte, and P. Sas. Hybrid finite element - wave based method for acoustic problems. Computer Assisted Mechanics and Engineering Sciences, 11:375-390, 2003.

[71] I. Schur. Über die Potenzreihen die im Innern des Einheitkreises beschränkt sind, I. Journal für die Reine und Angewandte Mathematik, 147:205-232, 1917.

[72] I. Schur. Über die Potenzreihen die im Innern des Einheitkreises beschränkt sind, II. Journal für die Reine und Angewandte Mathematik, 148:122-145, 1918.

[73] MATLAB. Solve systems of linear equations Ax=B for $\mathrm{x}$. http://bit.ly/2podzKR. Online; accessed August 21 st 2017.

[74] J. F. Allard and N. Atalla. Propagation of Sound in Porous Media: Modeling Sound Absorbing Materials. John Wiley \& Sons, West Sussex, United Kingdom, 2nd edition, 2009.

[75] P. Bouillard and R. Ihlenburg. Error estimation and adaptivity for the finite element method in acoustics: 2D and 3D applications. Computer Methods in Applied Mechanics and Engineering, 176:147-163, 1999.

[76] N.-E. Hörlin, M. Nordström, and P. Göransson. A 3-D hierarchical FE formulation of Biot's equations for elasto-acoustic modelling of porous media. Journal of Sound and Vibration, 245:633-652, 2001.

[77] J.-P. Groby, A. Duclos, O. Dazel, L. Boeckx, and L. Kelders. Enhancing absorption coefficient of a backed rigid frame porous layer by embedding circular periodic inclusions. The Journal of the Acoustical Society of America, 130:3771-3780, 2011.

[78] B. Van Genechten, D. Vandepitte, and W. Desmet. A direct hybrid finite element - Wave based modelling technique for efficient coupled vibro-acoustic analysis. Computer Methods in Applied Mechanics and Engineering, 200:742-764, 2011.

[79] S. Jonckheere, E. Deckers, B. Van Genechten, D. Vandepitte, and W. Desmet. A direct hybrid Finite Element - Wave Based Method for steady-state analysis of acoustic cavities with poro-elastic damping layers using the coupled Helmholtz-Biot equations. Computer Methods in Applied Mechanics and Engineering, 263:144-157, 2013. 